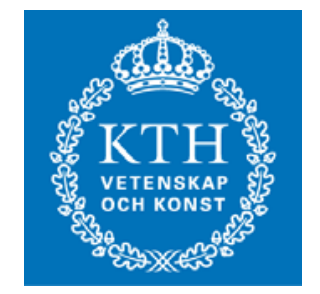

\title{
NOVEL ROOM TEMPERATURE FERROMAGNETIC SEMICONDUCTORS
}

\section{Amita Gupta}

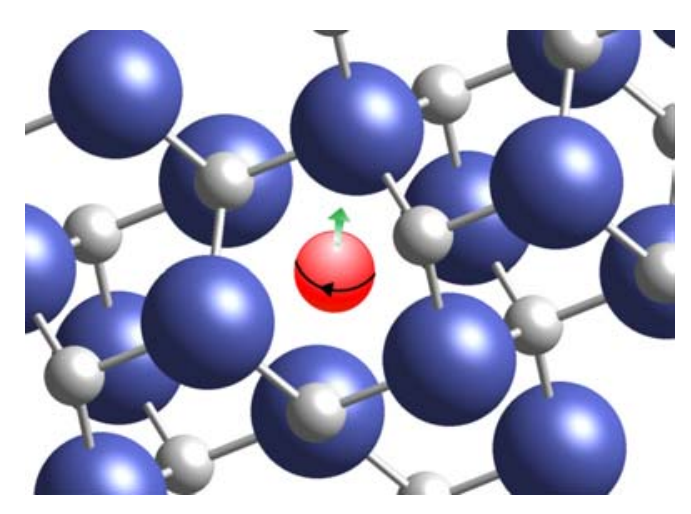

Stockholm, June 2004

Doctoral Dissertation

Royal Institute of Technology

Dept of Materials Science and Engineering

Division of Engineering Materials Physiscs

Fakultetsopponent: Dr. Vivek Mehrotra, Manager, Emerging Technologies, Rockwell Scientific, USA

Akademisk avhandling som med tillstånd av Kungl. Tekniska Högskolan framlägges till offentlig granskning för avläggande av teknologie doktorsexamen

måndagen den 14 June, kl. 10.00 i Kollegiesalen, Kungl. Tekniska Högskolan. 


\title{
Surprises bring in new challenges!
}

\begin{abstract}
In today's information world, bits of data are processed by semiconductor chips, and stored in the magnetic disk drives. But tomorrow's information technology may see magnetism (spin) and semiconductivity (charge) combined in one 'spintronic' device that exploits both charge and 'spin' to carry data (the best of two worlds). Spintronic devices such as spin valve transistors, spin light emitting diodes, non-volatile memory, logic devices, optical isolators and ultra-fast optical switches are some of the areas of interest for introducing the ferromagnetic properties at room temperature in a semiconductor to make it multifunctional. The potential advantages of such spintronic devices will be higher speed, greater efficiency, and better stability at a reduced power consumption.
\end{abstract}

This Thesis contains two main topics: In-depth understanding of magnetism in Mn doped $\mathrm{ZnO}$, and our search and identification of at least six new above room temperature ferromagnetic semiconductors. Both complex doped $\mathrm{ZnO}$ based new materials, as well as a number of nonoxides like phosphides, and sulfides suitably doped with $\mathrm{Mn}$ or $\mathrm{Cu}$ are shown to give rise to ferromagnetism above room temperature. Some of the highlights of this work are discovery of room temperature ferromagnetism in

- ZnO:Mn (paper in Nature Materials, Oct issue, 2003)

- ZnO doped with $\mathrm{Cu}$ (containing no magnetic elements in it!)

- GaP doped with $\mathrm{Cu}$ (again containing no magnetic elements in it!)

- Enhancement of Magnetization by Cu co-doping in $\mathrm{ZnO}: \mathrm{Mn}$

- CdS doped with Mn, and a few others not reported in this thesis.

We discuss in detail the first observation of ferromagnetism above room temperature in the form of powder, bulk pellets, in 2-3 $\mu \mathrm{m}$ thick transparent pulsed laser deposited films of the $\mathrm{Mn}(<4$ at.\% ) doped $\mathrm{ZnO}$. High-resolution transmission electron microscopy (HRTEM) and electron energy loss spectroscopy (EELS) spectra recorded from 2 to 200nm areas showed homogeneous distribution of $\mathrm{Mn}$ substituting for $\mathrm{Zn} \mathrm{a} 2^{+}$state in the $\mathrm{ZnO}$ lattice. Ferromagnetic Resonance (FMR) technique is used to confirm the existence of ferromagnetic ordering at temperatures as high as $425 \mathrm{~K}$. The ab initio calculations were found to be consistent with the observation of ferromagnetism arising from fully polarized $\mathrm{Mn} 2^{+}$state. The key to observed room temperature ferromagnetism in this system is the low temperature processing, which prevents formation of clusters, secondary phases and the host $\mathrm{ZnO}$ from becoming n-type. The electronic structure of the same $\mathrm{Mn}$ doped $\mathrm{ZnO}$ thin films studied using XAS, XES and RIXS. revealed a strong hybridization between Mn 3d and O 2p states, which is an important characterstic of a Dilute magnetic Semiconductor (DMS).

It is shown that the various processing conditions like sintering temperature, dopant concentration and the properties of precursors used for making of DMS have a great influence on the final properties. Use of various experimental techniques to verify the physical properties, and to understand the mechanism involved to give rise to ferromagnetism is presented. Methods to improve the magnetic moment in $\mathrm{Mn}$ doped $\mathrm{ZnO}$ are also described. New promising DMS materials (such as $\mathrm{Cu}$ doped $\mathrm{ZnO}$ are explored).

The demonstrated new capability to fabricate powder, pellets, and thin films of room temperature ferromagnetic semiconductors thus makes possible the realization of a wide range of complex elements for a variety of new multifunctional phenomena related to Spintronic devices as well as magneto-optic components. 


\section{Introduction}

Devices continue to diminish in size in order to achieve higher speeds. As this shrinkage occurs, the design parameters are impacted in such a way that the materials in current use are pushed to their limits [1]. Another alternative would be to render the individual components multifunctional. Several long-term alternative concepts are being investigated that would reduce the device size, power consumption, and exploit multifunctional properties of materials. One of the hot topics today is to use the spin of electrons, holes, nuclei, or ions to gain new functionalities in both analog and digital electronics. The charge, mass and spin of electrons form the foundation of present information technology. The integrated circuits and the high frequency devices made of semiconductors, used for information processing, use only the charge of electrons while the storage of information is done by magnetic recording using spin of electrons in a ferromagnetic metal $[2,3]$. But tomorrow's information technology may see magnetism (spin), and semiconductivity (charge) combined in one device that exploits both charge and 'spin' to process, and store the information. We may then be able to use the capability of mass storage and processing of information in the same device. Such a device will be called as "Spintronic device" [4]. This could not be realized so far because the semiconductors currently used in integrated circuits, transistors and lasers, such as silicon and gallium arsenide are nonmagnetic. Moreover, in order to have a useful difference in the energy between the two possible electron spin orientations (up and down), the magnetic fields that would have to be applied are too high for everyday use [5]. There are semiconductors, which have a periodic array of magnetic elements eg. Europium, chalcogenides and semiconducting spinels but their crystal structure is quite different from that of Si and GaAs. Also their crystal growth is very difficult. Hence they are not ideal for spintronic applications [5].

One of the approaches to drive a semiconductor ferromagnetic is to introduce magnetic ions like $\mathrm{Mn}, \mathrm{Cr}, \mathrm{Co}$ and $\mathrm{Fe}$ into non-magnetic semiconductors. In these ferromagnetic semiconductors, a part of the lattice is made up of substitutional magnetic atoms. Hence they are called diluted magnetic semiconductors (DMS) [6]. In recent years there has been an extensive research towards introducing ferromagnetic property at room temperature in semiconductors to realize a new class of spintronic devices such as spin valves, transistors, spin light emitting diodes, magnetic sensors, non-volatile memory, logic devices, optical isolators and ultra-fast optical switches. The potential advantages of 
spintronic devices will be higher speed, greater efficiency, and better stability, in addition to the low energy required to flip a spin [4].

Most of the past work on DMS has focussed on (Ga,Mn)As and (In,Mn)As. But the highest reported Curie Temperature (Tc) in the single phase samples grown by MBE range from $\sim 35$ to $172 \mathrm{~K}[7,8]$. This quest for a room temperature Ferromagnetic Semiconductor, gained momentum, following a theoretical prediction by Dietl et al., that $\mathrm{ZnO}$ and $\mathrm{GaN}$ would exhibit ferromagnetism above room temperature on doping with Mn, provided that the hole density is sufficiently high [9]. Several researchers have since then reported observation of room temperature ferromagnetism in doped semiconductors. A considerable attention has been paid to semiconductors doped with ferromagnetic metals ( $\mathrm{Co}, \mathrm{Fe}$, and $\mathrm{Ni}$ ). In these types of systems, the fundamental issue of much concern is that the ferromagnetic ordering could be a result of metal precipitates e.g., $\mathrm{Co}$ in $\mathrm{Co}$ doped $\mathrm{TiO}_{2}$ and $\mathrm{Co}$ doped $\mathrm{ZnO}[10,11,12]$. Moreover, a definite picture regarding the actual mechanism of ferromagnetic ordering in these systems has not been established.

A $\mathrm{ZnO}$ based DMS would be very promising because of its widespread applications in electronic devices, such as transparent conductors, gas sensors, varistors, surface acoustic wave devices, optical wave guides, acoustooptic modulators/deflectors, ultra violet laser source, and detectors [13]. Out of all the transition metals (TM), the doping of $\mathrm{Mn}$ in $\mathrm{ZnO}$ is most favourable because Mn has the highest possible magnetic moment [14] and also the first half of the d-band is full, creating a stable fully polarized state. The theoretical studies on Mn doped $\mathrm{ZnO}$ also proved its novelties in the fabrication of room temperature spintronic devices. $\mathrm{A} \mathrm{T}_{\mathrm{c}}$ higher than $300 \mathrm{~K}$ for p-type $\mathrm{ZnO}$ has been predicted theoretically, but this has not been realized experimentally. Moreover, $\mathrm{Mn}$ is known to be anitiferromagnetic [15], which makes this system more clean in terms of metal precipitate induced ferromagnetism, which is the subject of great controversies in DMS. Despite uncertainity in the mechanism of ferromagnetism in doped semiconductors, and the fact that the obtained magnetisation is lower than the theoretically predicted value in most of the reports appearing in literature, the results reported thus far, provide a pathway for exploring the Mn doped DMS. It is however, imperative to understand the phenomenon and the factors affecting the magnetisation value in order to realize commercially applicable devices. 


\subsection{Objectives}

The main aim of the present thesis is to show that it is indeed possible to obtain room temperature ferromagnetic semiconductors by controlling the process parameters. Theoretical prediction of room temperature ferromagnetism in $\mathrm{Mn}$ doped $\mathrm{ZnO}$ could be realized experimentally. Its is shown that various processing conditions like sintering temperature, dopant concentration and the properties of precursors used for making of DMS have a great influence on the final properties of the material. Use of various experimental techniques to verify the physical properties, and to understand the mechanism is demonstrated. Methods to improve the magnetic moment are also described. Other promising DMS materials, that is, $\mathrm{Cu}$ doped $\mathrm{ZnO}$ and $\mathrm{GaP}$ doped with $\mathrm{Cu}$ are investigated. We have also persued an active search for new materials that satisfies the criteria of above room temperature ferromagnetism, with a capability to control the carrier type and concentrations. 


\section{Spintronics}

Best of both worlds!

Electrons, like all fundamental particles, have a property called spin which can be orientated in one direction or the other - called 'spin-up' or 'spin-down' - like a top spinning anticlockwise or clockwise [4]. When electron spins are aligned (ie all spin-up or all spindown) and interact they can create a large-scale net magnetic moment as seen in magnetic materials like iron and cobalt. Magnetism is an intrinsic physical property associated with the spins of electrons in a material. The directional property of magnetism is already exploited in recording devices such as computer hard disks [16]. Data are recorded and stored as tiny areas of iron or chromium oxide magnetised with the direction of magnetically ordered elements, controlled and easily manipulated to be in-plane or out-of-plane (a basis for the so called perpendicular recording) [17]

Conventional electronic devices rely on the transport of electrical charge carriers electrons - in a semiconductor such as silicon. The infomation processing is done by utilizing circuits using transistors that work by transfer of electrons [18]. A schematic of a conventional metal oxide field effect transistor is shown in Fig. 1. A Field-Effect-Transistor (MOSFET) consists of a source and a drain, two highly conducting n-type semiconductor regions, which are isolated from the p-type substrate by reversed-biased p-n diodes. A metal (or poly-crystalline) gate covers the region between source and drain (Fig. 1). The flow of electrons from the source to the drain is controlled by the voltage applied to the gate.

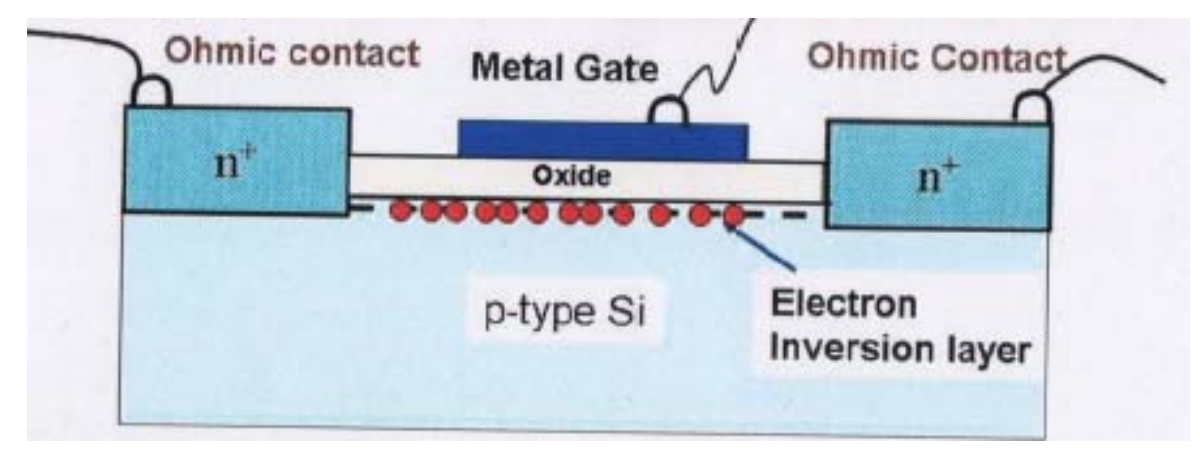

Fig-1 A metal oxide field effect transistor. Gate voltage changes electron density 
A conceptually similar structure shown in Fig. 1 was first proposed and patented by Lilienfeld and Heil in 1930, but was not successfully demonstrated until 1960. Now, with the advancement in technology, the devices are diminishing in size and increasing in speed. The number of transistors on an IC chip, doubles every 18 months-following the so called Moore's law [1,4]. However, Moore's law will subsequently lose momentum as the size of individual bit approaches dimensions of an atom. For this reason and to provide multifunctionality to the devices, investigators are trying to combine both, charge and spin degrees of freedom of electrons, to create a remarkable new generation of devices which will be smaller, more versatile and more robust than those currently making up silicon chips and circuit elements. Thus emerges a new field of electronics called spin electronics or spintronics $[2,4]$.

The advantage of spin over charge is that spin can be easily manipulated by externally applied magnetic fields, a property already in use in magnetic storage technology. Another significant property of spin is its long coherence, or relaxation, time (nanoseconds, compared to tens of femtoseconds during which electron momentum decays) - once created it tends to stay that way for a long time, unlike charge states, which are easily destroyed by scattering or collision with defects, impurities or recombination [4]. These characteristics open the possibility of developing devices that could be much smaller, consume less power and will be more powerful for certain types of computations which is not possible with electron-chargebased systems. It is widely expected that new functionalities for electronics and photonics can be derived if injection, transfer and detection of carrier spin can be controlled over room temperature [1].

\subsection{Spintronic devices}

Spintronic devices came into action after the discovery of powerful effect called "Gaint magnetoresistance (GMR)" in 1988 by French and German physicists [19]. It results from subtle electron-spin effects in ultra-thin 'multilayers' of magnetic materials, which cause huge changes in their electrical resistance when a magnetic field is applied. It is a sandwich structure with alternating layers of magnetic and nonmagnetic metal (Fig-2). Depending upon the relative orientations of the magnetizations in the magnetic layers, the electrical resistance changes from small (parallel magnetizations) to large (antiparallel magnetizations). The magnitude of this change is two order of magnitude larger than is possible with conventional materials, hence the name "giant magnetoresistance"[4]. 


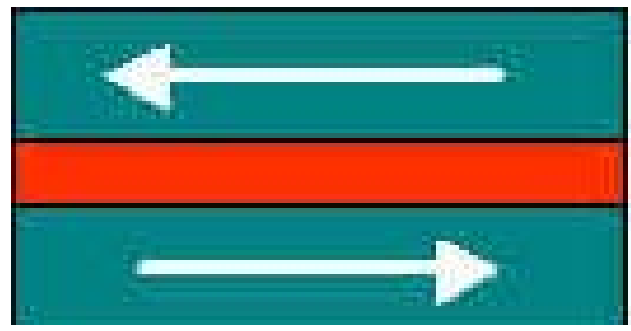

Ferromagnet

Spacer-nonmagnetic

Ferromagnet

Fig-2 A GMR sandwich structure. Consisting of alternating magnetic and nonmagnetic metal layers. Layers.

IBM soon realised that read heads incorporating GMR materials would be able to sense much smaller magnetic fields, allowing the storage capacity of a hard disk to increase from 1 to 20 gigabits [20] In 1997 IBM launched GMR read heads, into the market. Physicists have been quick to see the further possibilities of spin valves. Not only they are highly sensitive magnetic field sensors, they can also be made to act as switches by flipping the magnetisation in one of the layers. This allows information to be stored as $0 \mathrm{~s}$ and $1 \mathrm{~s}$ (magnetisations of the layers parallel or antiparallel) as in a conventional transistor memory device. An obvious application is a magnetic version of a random access memory (MRAM) device of the kind used in computers [21]. The advantage of magnetic random access memory (MRAM) is that it is 'non-volatile' - information is not lost when the system is switched off. MRAM devices would be smaller, faster, cheaper, use less power and would be much more robust in extreme conditions such as high temperature, or high-level radiation or interference. The US electronics company Honeywell has already shown that arrays of linked MRAMS could be made to work.

MRAM is rapidly developing as a technology because it can allow quantum computing by use of spin of individual particles to process information. Such an information bearing particle is known as a quantum bit or qubit. It is amazing to know that only 34 qubits are required to represent the total amount of information stored in a $10 \mathrm{~Gb}$ hardrive [1]! With the exception of MRAM, none of the spintronic devices such as spin based light emitting diodes (spin LEDs), resonant tunneling diodes (spin RTDs), field effect transistors (spin FETs), and spin based single electron devices based on quantum dot arrays can be fabricated without the ability to generate, maintain, and propagate long lived spins in a semiconductor $[1]$. 


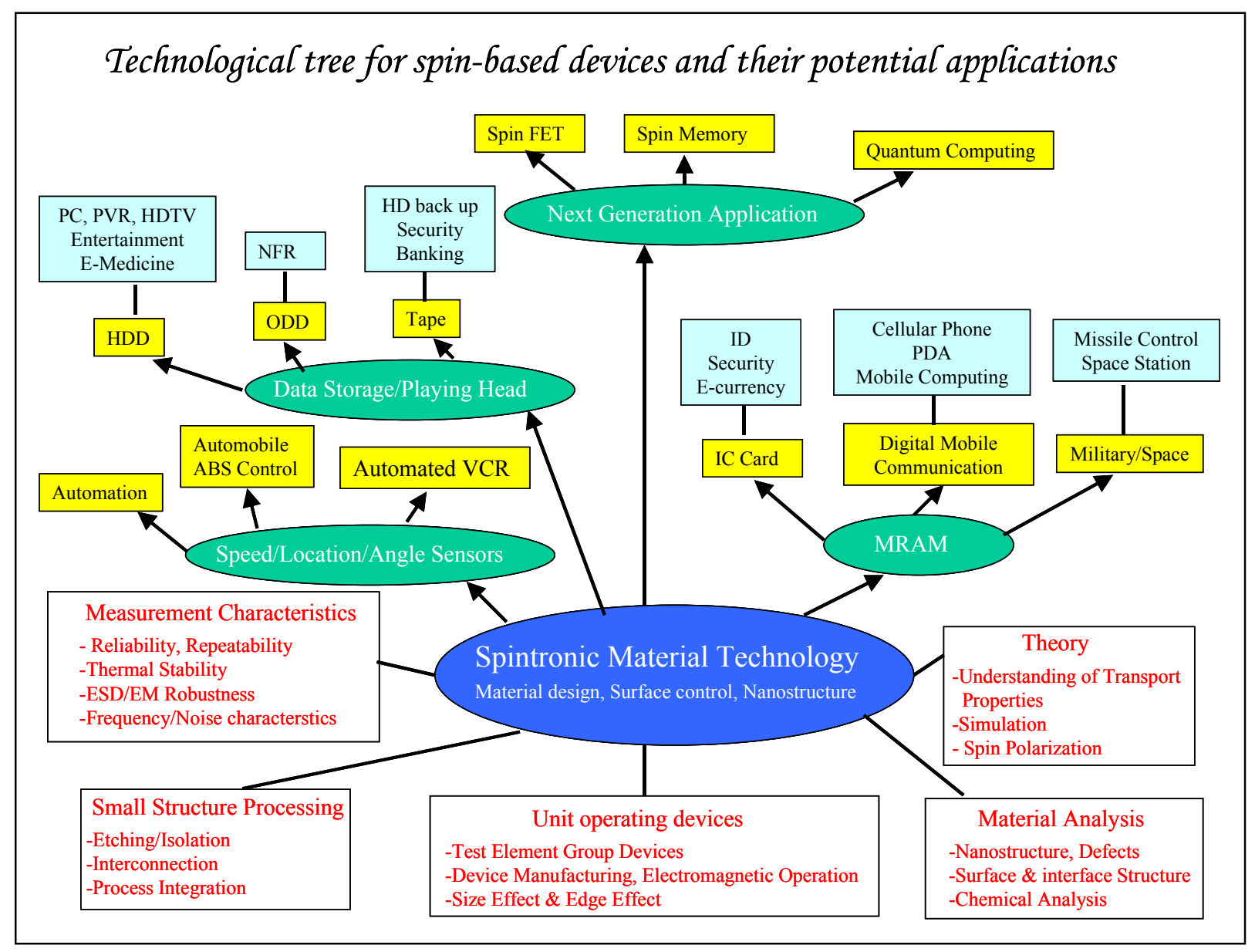

Fig. 3 A technology tree for Spin-based devices [3]

All spintronic or more specifically semiconductor spintronic devices act according to the simple scheme: (1) information is stored (written) into spins as a particular spin orientation (up or down), (2) the spins, being attached to mobile electrons, carry the information along a wire, and (3) the information is read at a terminal [22]. The long coherence time of spin makes spintronic devices particularly attractive for memory storage and magnetic sensors applications, and, potentially for quantum computing where electron spin would represent a bit (called qubit) of information [18]. The basic idea behind semiconductor based spintronics is to add the characteristics of magnetic devices to existing devices such as light-emitting diodes and field effect transistors [4]. This would lead to technologies such as memory and microprocessor functions integrated on the same chip, magnetic devices with gain and integrated sensors with on-chip signal processing and off-chip optical communications. A technology tree which summarizes the spin-based devices is shown is shown in Fig.3. 


\subsubsection{Spin-FET:}

The first appearance of the idea of a spintronic device was the Spin-FET proposed by Datta et al. in 1990 [23]. In this proposal, taking into account similarity of electrical and optical systems, they proposed that in direct analogy with an electro-optic modulator (in which voltage variability of the polarization factors of the electro-optic material are used to control the output power), a small band gap compound semiconductor field effect transistor with polarizing ferromagnetic/semiconductor source and drain contacts, can enjoy the current modulation due to spin-orbit coupling. The device of Datta/Das proposal is demonstrated in Fig. 4

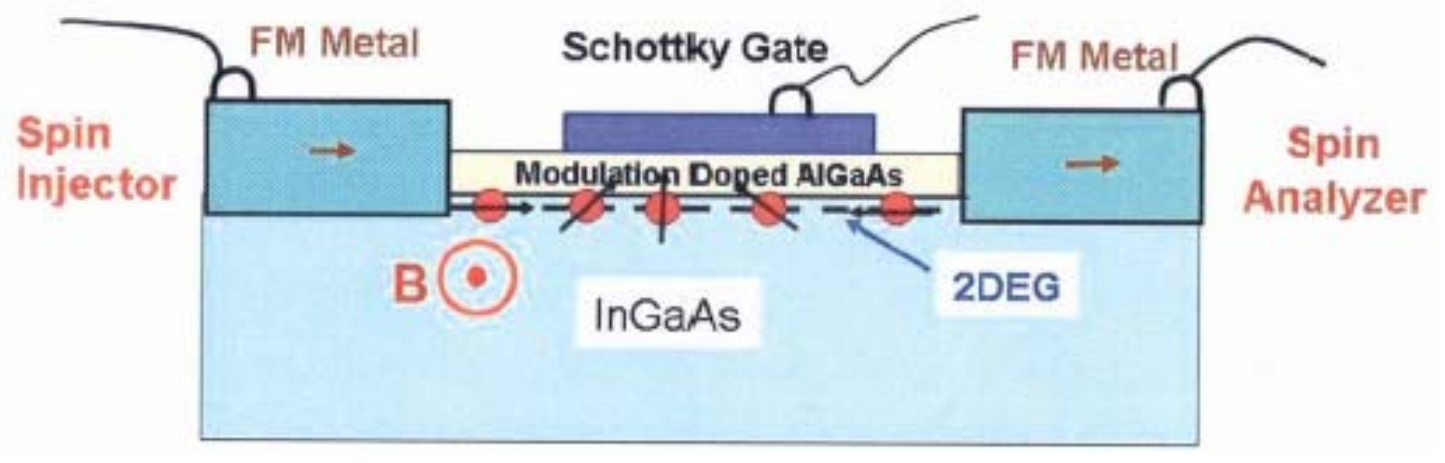

Fig. 4 Datta/Das Spin FET [23]

In the structure shown in Fig. 4, the ferromagnetic contacts are used to preferentially inject and detect specific spin orientations. For this kind of source/drain (or polarizer/analyzer) contact, the density of states at the fermi level for electrons of one spin greatly exceeds that of the other one; this way the contact preferentially injects and detects electron of a single spin.

The operation of Spin-FET is same as in a conventional charge controlled FET. In a spin FET, application of a relatively low gate voltage causes an interaction between the electric field and the spin precession of the carriers. If this is sufficient to put the spin orientation of the carriers out of alignment with the drain contact, then the current is effectively shut off and this can occur at much lower biases than is needed to shut off the current in a charge-controlled FET. 


\subsubsection{Spin LED:}

Efficient spin injection from the ferromagnetic metals and dilute magnetic semiconductors into the semiconductor is the fundamental requirement of the semiconductor based spintronic devices. Spin-LED is used to measure the spin

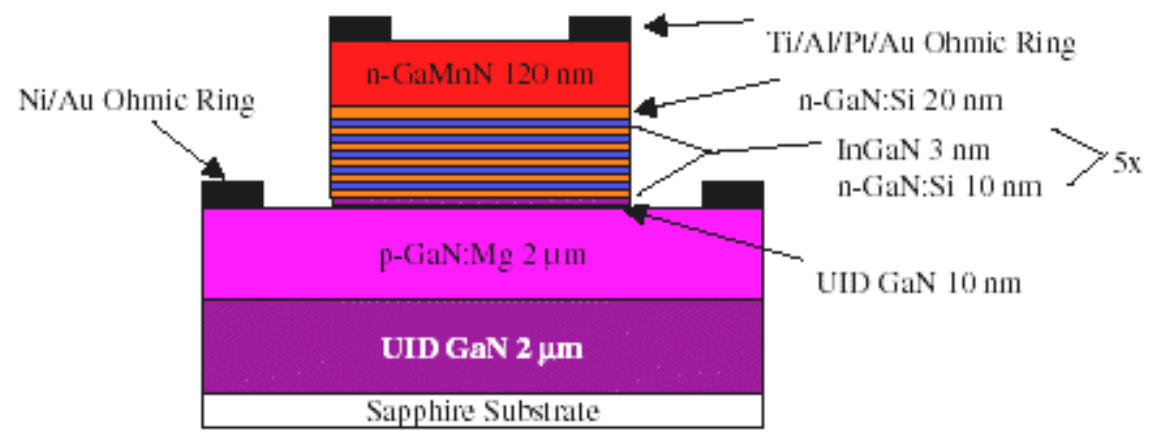

Fig. 5 A schematic of a spin LED [24]

injection efficiency into materials. In a spin LED circularly polarized light is emitted after the recombination of spin-polarized carriers that are electrically injected into a semiconductor heterostructure. Such a device should allow us to modulate the polarization of the light emitted by the spin LED by application of an external magnetic field. The most straightforward approach to achieve this goal would be to implant Mn into the top contact pGaN layer of the standard GaN/InGaN LED. Fig-3 shows a schematic of a spin LED.

\subsubsection{Magnetic switch:}

Recently, Ohono et al. [25] designed a magnetic switch, which has a layer of indium manganese arsenide, (In,Mn)As, material ed within a field-effect transistor . A positive voltage on the metallic gate electrode creates electric fields (shown by arrows in fig 6(a)) that repels holes causing the Mn moments to orient randomly. A negative voltage on the gate electrode creates electric fields that attract holes, causing the Mn magnetic moments to align. Shown below is a schematic of the magnetic switch- 


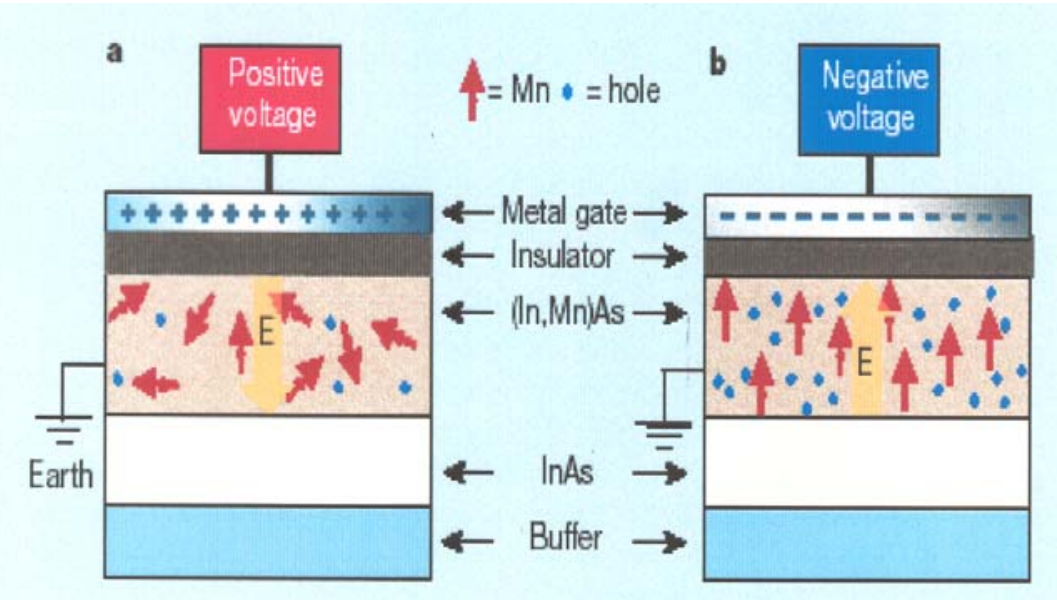

Fig. 6 A schematic of a magnetic switch [25]

In order to realize the spintronic devices in practice, ferromagnetic semiconductors functional at room temperature are needed. The search for suitable materials started in 1960s and several promising materials have been found since then. 


\section{History of Dilute Magnetic Semiconductors \\ A journey of thousand miles begins with a single step!}

The study of dilute magnetic semiconductors started in late 1960s [26,27] resulted in various exciting findings. Among the most extensively studied ferromagnetic semiconductors were Eu-Chalcogenides (e.g., EuSe, $\mathrm{EuS}, \mathrm{EuO}$ ) and $\mathrm{Cr}$-Chalcogenide spinels (e.g., $\mathrm{CdCr}_{2} \mathrm{Se}_{4}$, $\mathrm{CdCr}_{2} \mathrm{~S}_{4}$ ). In these magnetic semiconductors exchange interactions between the electrons in the semiconducting band and the localized electrons at the magnetic ions led to a number of interesting properties like red-shift of band gap when ferromagnetism sets in. Inspite of the numerous studies carried out on these materials, no practical applications have been realized. A low Curie temperature hampered the application and extreme difficulty in growing these crystals hindered further studies on these materials [27].

The next generation of DMS began in early 1980s with the appearance of dilute magnetic semiconductors based on II-VI compounds together with those based on IV-VI compounds [26]. The materials of the type $A_{1-x}^{I I} M n_{x} B^{V I}$ (e.g. $\mathrm{Cd}_{1-\mathrm{x}} \mathrm{Mn}{ }_{\mathrm{x}} \mathrm{Te}, \mathrm{Cd}_{1-\mathrm{x}} \mathrm{Mn}_{\mathrm{x}} \mathrm{Se}$, $\mathrm{Hg}_{1-\mathrm{x}} \mathrm{Mn}_{\mathrm{x}} \mathrm{Te}$ ) were of much interest because of several distinct reasons. Their ternary nature gave the possibility of tuning the lattice constant and band parameters by varying the composition of the material, thus making it possible to engineer their band gap [6]. The substitutional $\mathrm{Mn}$ atoms in $\mathrm{A}^{\mathrm{II}} \mathrm{B}^{\mathrm{VI}}$ lattice are also characterised by highly efficient electroluminiscence, which makes $A_{1-x}^{I I} M n_{x} B^{V I}$ alloys important in the context of optical flat panel display applications. Furthermore, the strong exchange interactions between sp band electrons and d electrons associated with $\mathrm{Mn}^{++}$resulted in interesting optical and electrical properties like giant Faraday rotation and magnetic and field induced metal-insulator transition [6].

In these materials the valency of cations matches that of common magnetic ions like Mn. This phenomenon makes this type of DMS relatively easy to make in bulk and thin epitaxial layers. Many fundamental studies were done on these systems but not much application could be realized except for optical isolators [26]. The magnetic interactions in these DMS is dominated by antiferromagnetic coupling of Mn spins, which results in paramagnetic, antiferromagnetic or spin glass behaviour [26]. A ferromagnetic DMS based on II-VI materials could not be realized until recently by A. Hauri et al. (Tc 2 K) [28]. Moreover, 
II-VI based DMS have been difficult to dope to create $\mathrm{p}$-and n-type, which made it difficult to study their transport properties and hence less attractive for applications [27].

Another group of materials, IV-VI materials (such as PbMnTe, nMnTe and GeMnTe) also attracted attention because of free carrier induced magnetism. Unlike II-VI DMS, these materials can be grown with higher concentration of free band carriers. Their magnetic properties can be controlled by modifying the carrier concentration through control of native defects. Story et al. demonstrated for the first time, effect of carrier concentration on magnetic properties of $\mathrm{PbSnMnTe}$ [29]. They reported a ferromagnetic ordering in this material up till $4 \mathrm{~K}$ and the $\mathrm{T}_{\mathrm{c}}$ could be increased by increasing the carrier concentration. However, giant moment often talked about in certain metallic alloys of transition metals cannot be formed in IV-VI alloys because of the diamagnetism of the host crystal. Recently ferromagnetic transition temperature as high as $100 \mathrm{~K}$ was reported in Fe doped GeTe films $[30]$.

The other class of semiconductors that is III-V semiconductors like GaAs and InAs had major markets in IR LEDs and lasers and magnetic sensors respectively [3]. Magnetism, especially ferromagnetism, has not been a part of activity in these semiconductors because of the lack of material technologies that would allow introduction of magnetic cooperative phenomena into III-V heterostructures [28]. The equilibrium solubility of transition metal atoms into these semiconductors is very low $\sim 10^{18} \mathrm{~cm}^{-3}$ [31] or less. Beyond a certain doping level, the surface segregation, and in extreme cases phase separation would occur and impede further incorporation of high concentration of magnetic ions into the crystals. Therefore, preparation of ternary alloys to form DMS of III-V semiconductors is an extremely difficult task. No DMS based on these materials was formed until Ohno et al. reported ferromagnetism in epitaxial films of InMnAs grown by Molecular Beam Epitaxy (MBE) in 1992 and GaMnAs (Tc $110 \mathrm{~K}$ ) in 1996 [32,33]. The low temperature used to grow a crystal by MBE prevents formation of a secondary phase. Since then both these systems have been investigated extensively in an attempt to increase the ferromagnetic transition temperature to more practical limits. Till date the highest reported Curie temperature in $\mathrm{Mn} \delta$-doped GaMnAs heterostructure is $\sim 172 \mathrm{~K}$ by Nazmul et al. [8].

Besides II-VI, IV-VI and III-V semiconductors based DMS, room temperature ferromagnetism was reported in a II-IV-V2 type chalcopyrite compound $\mathrm{CdGeP}_{2}$ doped with $\mathrm{Mn}$. [26]. It is easier to achieve p-type doping in ternary semiconductors with chalcopyrite type structure. Also II-IV-V 2 semiconductors are reported to have a higher carrier mobility, which is important for device applications [26]. 
Another important discovery was a group IV ferromagnetic semiconductor $\mathrm{Mn}_{\mathrm{x}} \mathrm{Ge}_{1-\mathrm{x}}$ by Park et al. in 2002 [34]. It followed a prediction based on mean field solution of Zener model that ferromagnetic (FM) order can be stabilised in group-IV semiconductors like $\mathrm{Si}, \mathrm{Ge}$ and $\mathrm{Si}_{1-\mathrm{x}} \mathrm{Ge}_{\mathrm{x}}$. The single crystal $\mathrm{Mn}_{\mathrm{x}} \mathrm{Ge}_{1-\mathrm{x}}$ films on Ge and GaAs (001) substrates have Curie temperatures varying linearly with the Mn concentration from $25 \mathrm{~K}$ to $116 \mathrm{~K}$. 


\section{State of the art}

Vision drive determination, eyes set on perfection!

Recent work on spintronics focuses on achieving practical magnetic ordering temperatures in technologically useful semiconductors. While the progress in synthesizing and controlling magnetic properties of III-arsenide semiconductors has been astounding, the reported Curie temperatures are too low $(\sim 172 \mathrm{~K})[8]$ to have a significant practical impact. A key development that focussed on wide band gap semiconductors as being the most promising for achieving high Curie temperatures, was the work of Dietl et al. They employed Zener model of ferromagnetism to predict $T_{c}$ values for several materials. The schematic showing the predicted Tc values is given below [9]

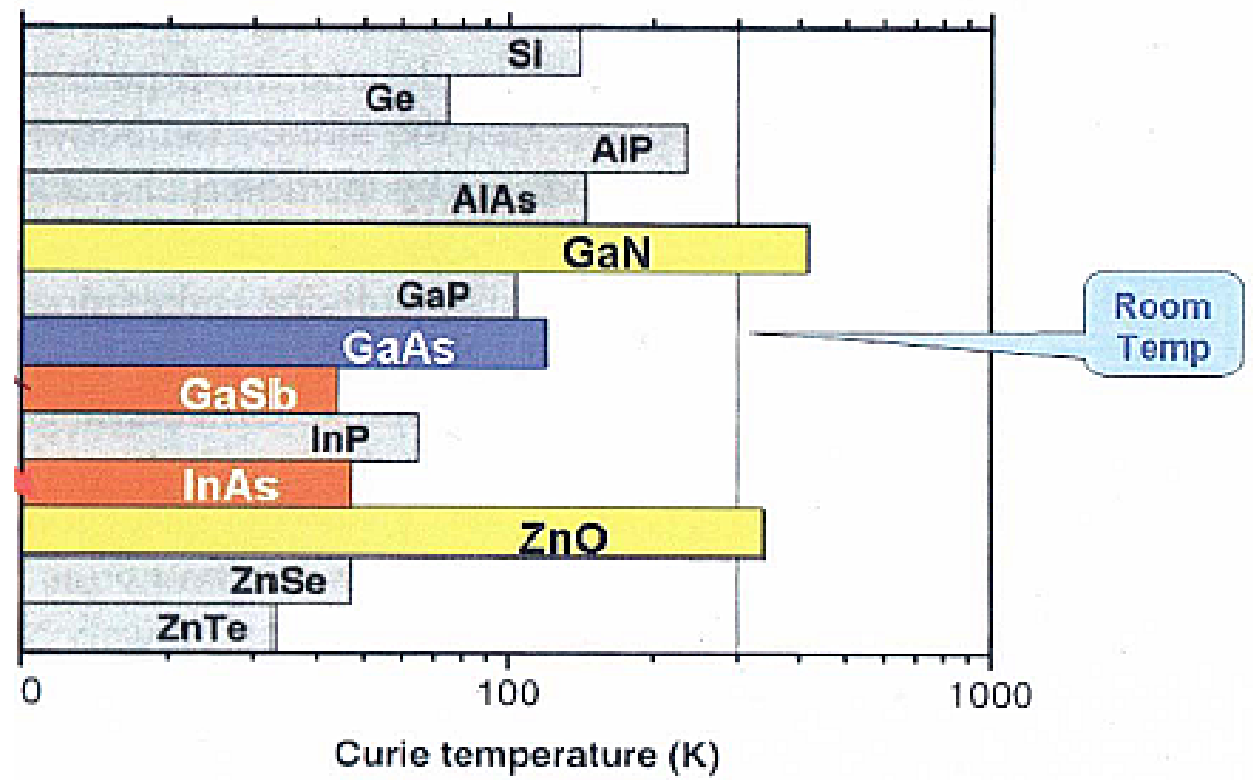

Fig-7Predicted Curie temperatures for doped semiconductors. [9]

The Tc exceeding room temperature for semiconductors such as $\mathrm{GaN}$ and $\mathrm{ZnO}$ containing $5 \% \mathrm{Mn}$ and a high hole concentration $\left(3.5 \times 10^{20} \mathrm{~cm}^{-3}\right)$ initiated intense research to realize a room temperature DMS based on wide band gap semiconductors. Tremendous progress has since been made both in realization of high quality epitaxial layers of DMS films and in the theory of ferromagnetism in these dilute magnetic semiconductors.

Several materials have been reported to have a room temperature ferromagnetic ordering. Some of them are listed here. 


\section{Material Curie temperature Submitted Published Ref.}

\begin{tabular}{|c|c|c|c|c|}
\hline $\mathrm{Cd}_{1-\mathrm{x}} \mathrm{Mn}_{\mathrm{x}} \mathrm{GeP}_{2}$ & $300 \mathrm{~K}$ & 000804 & 001001 & 26 \\
\hline $\mathrm{Ti}_{1-\mathrm{x}} \mathrm{Co}_{\mathrm{x}} \mathrm{O}_{2}$ & $>380 \mathrm{~K}$ & 000928 & 010202 & 35 \\
\hline $\mathrm{Zn}_{1-x} \mathrm{Co}_{x} \mathrm{O}$ & $300 \mathrm{~K}$ & 010201 & 010813 & 36 \\
\hline$Z n_{1-x} V_{x} O$ & $>350 \mathrm{~K}$ & 010823 & 0111 & 37 \\
\hline $\mathrm{Zn}_{1-\mathrm{x}} \mathrm{Mn}_{\mathrm{x}} \mathrm{Si}_{1-\mathrm{y}} \mathrm{Ge}_{\mathrm{y}} \mathrm{N}_{2}$ & $\sim 300 \mathrm{~K}$ & 011029 & 020815 & 38 \\
\hline $\mathrm{Zn}_{1-\mathrm{x}} \mathrm{Mn}_{\mathrm{x}} \mathrm{GeP}_{2}$ & $350 \mathrm{~K}$ & 011203 & 0203 & 39 \\
\hline $\mathrm{Ga}_{1-\mathrm{x}} \mathrm{Mn}_{\mathrm{x}} \mathrm{N}$ & $940 \mathrm{~K}$ & 011213 & $0211-0212$ & 40 \\
\hline $\mathrm{Zn}_{1-\mathrm{x}} \mathrm{Mn}_{\mathrm{x}} \mathrm{SnAs}_{2}$ & $329 \mathrm{~K}$ & 020123 & 0204 & 41 \\
\hline $\mathrm{Ga}_{1-\mathrm{x}} \mathrm{Mn}_{\mathrm{x}} \mathrm{P}$ & $330 \mathrm{~K}$ & 020128 & 020902 & 42 \\
\hline $\mathrm{Ga}_{1-\mathrm{x}} \mathrm{Cr}_{\mathrm{x}} \mathrm{N}$ & $>400 \mathrm{~K}$ & 020201 & 0204 & 43 \\
\hline $\mathrm{Ga}_{1-\mathrm{x}} \mathrm{Mn}_{\mathrm{x}} \mathrm{Sb}$ & $>400 \mathrm{~K}$ & 020327 & 020715 & 44 \\
\hline $\mathrm{Zn}_{1-x} \mathrm{Fe}_{x} \mathrm{O}$ & $550 \mathrm{~K}$ & 020502 & 021125 & 45 \\
\hline $\mathrm{Ga}_{1-\mathrm{x}} \mathrm{Gd}_{\mathrm{x}} \mathrm{N}$ & $>400 \mathrm{~K}$ & 020508 & 0206 & 46 \\
\hline $\mathrm{In}_{\mathrm{x}} \mathrm{Mn}_{1-\mathrm{x}} \mathrm{As}$ & $400 \mathrm{~K}$ & 020514 & 021203 & 47 \\
\hline $\mathrm{Al}_{1-\mathrm{x}} \mathrm{Cr}_{\mathrm{x}} \mathrm{N}$ & $>340 \mathrm{~K}$ & 020731 & 020923 & 48 \\
\hline $\mathrm{Zn}_{1-\mathrm{x}} \mathrm{Cr}_{\mathrm{x}} \mathrm{Te}$ & $\sim 300 \mathrm{~K}$ & 020829 & 030523 & 49 \\
\hline $\mathrm{Ga}_{1-\mathrm{x}} \mathrm{Co}_{\mathrm{x}} \mathrm{N}$ & $>320 \mathrm{~K}$ & 020919 & 030415 & 50 \\
\hline $\mathrm{Ti}_{1-\mathrm{x}} \mathrm{Fe}_{\mathrm{x}} \mathrm{O}_{2}$ & $\sim 300 \mathrm{~K}$ & 020930 & 0302 & 51 \\
\hline $\mathrm{Sn}_{1-\mathrm{x}} \mathrm{Co}_{\mathrm{x}} \mathrm{O}_{2}$ & $650 \mathrm{~K}$ & 030116 & 030815 & 52 \\
\hline $\mathrm{Zn}_{1-\mathrm{x}} \mathrm{Mn}_{\mathrm{x}} \mathrm{O}$ & $>425 \mathrm{~K}$ & 030219 & 030921 & 53 \\
\hline $\mathrm{Zn}_{1-x} \mathrm{Ni} i_{x} \mathrm{O}$ & $>350 \mathrm{~K}$ & 030729 & 030910 & 54 \\
\hline
\end{tabular}




\section{Mechanisms of ferromagnetism}

It is an important challenge of materials science to understand the ferromagnetism in magnetic semiconductors and to develop functional semiconductor systems with the Curie temperatures $T_{\mathrm{C}}$ exceeding comfortably the room temperature.

The fact that the DMS are ferromagnetic independent of their weakly metallic or strongly insulating nature implies a robust character for the underlying mechanism leading to the long range magnetic order in these systems. Clearly the ferromagnetic mechanism should not depend crucially on the carrier system being "free" valence band holes since the strongly insulating DMS systems do not have any free holes. The currently accepted picture for DMS ferromagnetism is that it is the local antiferromagnetic coupling between the carriers (i.e., holes in GaMnAs) and the Mn magnetic moments that leads to long range ferromagnetic ordering of Mn local moments. The carrier system also becomes spin-polarized in the process with the carrier magnetic moment directed against the Mn magnetic ordering by virtue of the antiferromagnetic hole-Mn coupling, but the total magnetic moment of the spin polarized carriers is extremely small since $n_{c}<<n_{i}$ and $|S|>|s|$ where $S$ and s are respectively the Mn and the hole spin and $n_{c}$ and $n_{i}$ are carrier and local moment density respectively. The relevant DMS effective magnetic Hamiltonian can be written as [55]

$$
H_{M}=\int d^{3} r J(r) S(r) . s(r)
$$

where S(r) and s(r) are respectively the Mn and hole spin densities. The coupling J(r) between Mn local moments and hole spins can, in principle, be ferromagnetic $(\mathrm{J}<0)$ or antiferromagnetic $(\mathrm{J}>0)$, but the effective interaction between the Mn local moments mediated by the holes (through HM) is always ferromagnetic. The magnitude of $\mathrm{J}$ must come from a first principles band structure calculation or from experiments. Since the Mn moments are localized at specific lattice sites, a conventional form of equation 1 would be [55]

$$
H_{M}=J \sum \int d^{3} r \delta\left(r-R_{i}\right) S_{i} s(r)
$$

where the sum over $\mathrm{i}$ goes over the Mn sites in the GaAs lattice, and we have assumed the exchange coupling $\mathrm{J}$ to be a constant. The JSi-s local exchange coupling defined in Eq. 2) is sometimes referred to as the Zener model (or the $\mathrm{s}-\mathrm{d}$ coupling although in the context of GaMnAs, where the carriers are holes, the coupling is more like an $\mathrm{p}-\mathrm{d}$ exchange coupling between Mn d levels and the $\mathrm{p}$ valence band ( $\mathrm{p}-\mathrm{d}$ hybridization) 
The DMS systems provide an interesting novel regime for studying $\mathrm{H}_{M}$, namely the regime of low carrier density $n_{i}>n_{c}$ which is normally the case for DMS. The simplest way to understand DMS ferromagnetism on a qualitative level is to neglect all disorder effects, and assume that the system can be thought of as a collection of local moments of density, $n_{i}$, interacting with itinerant holes of density $n_{c}$.

In the case of Mn-based IV-VI, III-V and II-VI, diluted magnetic semiconductors (DMS) the ferromagnetism can be observed provided that the hole concentration is sufficiently high. According to experimental and theoretical results, the ferromagnetic order in Mn-based DMS is mediated by carriers residing in relatively wide valence bands. In the case of III-V and II-VI DMS, the holes are coupled to the localised spins via a strong, symmetry allowed, antiferromagnetic p-d interaction. However, the $\mathrm{d}$ levels of transition metals other than Mn, reside in the band gap of III-V and II-VI compounds. In such a situation the double exchange may constitute the dominant mechanism of spin-spin interactions [56].

Obviously, magnetic properties of a semiconductor containing magnetic ion will depend on energetic positions of states derived from magnetic shells as well as on their interactions with the host bands. Furthermore, the energy of the magnetic levels in respect to host bands together with the on-site correlation energy $U$ determine whether the magnetic ion act as a dopant and how its charge and spin state depends on the presence of other impurities [56].

Mn is divalent in II-VI compounds, and assumes the high spin $\mathrm{d}^{5}$ configuration characterized by $\mathrm{S}=5 / 2$ and $\mathrm{g}=2$ [56] .The Mn ions neither introduce nor bind carriers, but give rise to the presence of the localized spin in II-VI DMS. The spin dependent hybridization between anion $\mathrm{p}$ and $\mathrm{Mn} \mathrm{d}$ states leads to the superexchange, a short-range antiferromagnetic coupling among the Mn moments. So for low carrier densities II-VI DMS are paramagnets but having all near neighbours Mn-Mn pairs antiferromagnetically blocked. However, the antiferromagnetic superexchange can be overcompensated by ferromagnetic interactions mediated by band holes. Since the electronic states in doped semiconductors undergo dramatic changes as a function of impurity concentration, hence the hole states, and possibly hole mediated exchange mechanisms, may undergo changes as a function of $\mathrm{Mn}$ concentration, $x$, and the concentration of acceptors $\mathrm{N}_{\mathrm{A}}$ and compensating donors $\mathrm{N}_{\mathrm{D}}$. The evolution of electronic states in doped semiconductors is governed by the ratio of the average distance between the carriers $r_{c}, r c=(3 / 4 \pi p)^{1 / 3}, p=x_{o}-N_{d}$, to the effective impurity Bohr radius $a_{B}$ 
The large exchange energy, $\beta$ No and high density of states, in case of systems with hole mediated ferromagnetic ordering, the ferromagnetic transition temperature $T_{c}$ can be high e.g $\beta$ No $=-1.2 \mathrm{eV}$ [57] in GaMnAs gave a $\mathrm{T}_{\mathrm{c}}$ of $110 \mathrm{~K}$ for $\mathrm{Mn}$ concentration of $3.5 \mathrm{at} \%$ containing $3.5 \times 10^{20}$ holes per $\mathrm{cm}^{3}$. But in case of II-VI DMS it is essential to take into account the magnetically inert nearest Mn-Mn pairs. Such pairs not only lower the effective Mn concentration but also Tc and $\mathrm{p}$. Hence presence of holes is essential to obtain ferromagnetic ordering in II-VI semiconductors. To accomplish this, additional acceptor dopant can be added [56]. 


\section{Review of some widely studied materials.}

6.1.1 (Ga,Mn)As

The application of non-equilibrium methods to grow III-V-based DMS was rewarded by successful molecular beam epitaxy (MBE) of uniform (In, Mn)As films on GaAs substrates by Munekata et al. in 1989 [31]. Subsequent discovery of the hole-induced ferromagnetic ordering in p-type (In, Mn)As by Ohno et al. in 1992 [32] encouraged researchers to investigate GaAs-based system (De Boeck et al. 1996) [58]. A successful growth of ferromagnetic ( $\mathrm{Ga}, \mathrm{Mn}$ )As by (Ohno et al. in 1996 [33] opened up possibility of room temperature DMS using III-V semiconductors which were neglected because of difficulty of doping with transition metals. Currently, a number of groups are working on the MBE growth of $(\mathrm{Ga}, \mathrm{Mn}) \mathrm{As}$ and related heterostructures to advance the understanding of this new class of materials [59-64].

GaAs is a well-understood and extensively studied III-IV compound, with a large band gap of $1.43 \mathrm{eV}$ as compared to $0.36 \mathrm{eV}$ for InAs. Hence GaAs along with its alloys is used in room temperature operation of electronic devices as well as optical radiation devices in the near infrared region, which is widely used in short-range optical communications.

Ohno et al. [33] grew $\mathrm{Ga}_{1-\mathrm{x}} \mathrm{Mn}_{\mathrm{x}} \mathrm{As}$ films by solid source MBE on semi-insulating (001) GaAs substrate ( $\mathrm{x}=0.015$ to 0.07 ). The lattice constant of the films was studied by Xray diffraction, and found to increase with the increase in Mn concentration. The XRD patterns did not show presence of any secondary phase like MnAs in the films. Fig. 8 shows the magnetic field dependence of the magnetization for sample with $\mathrm{x}=0.035$ at $5 \mathrm{~K}$. A clear square hysteresis indicated well-ordered ferromagnetic structure in the film. 


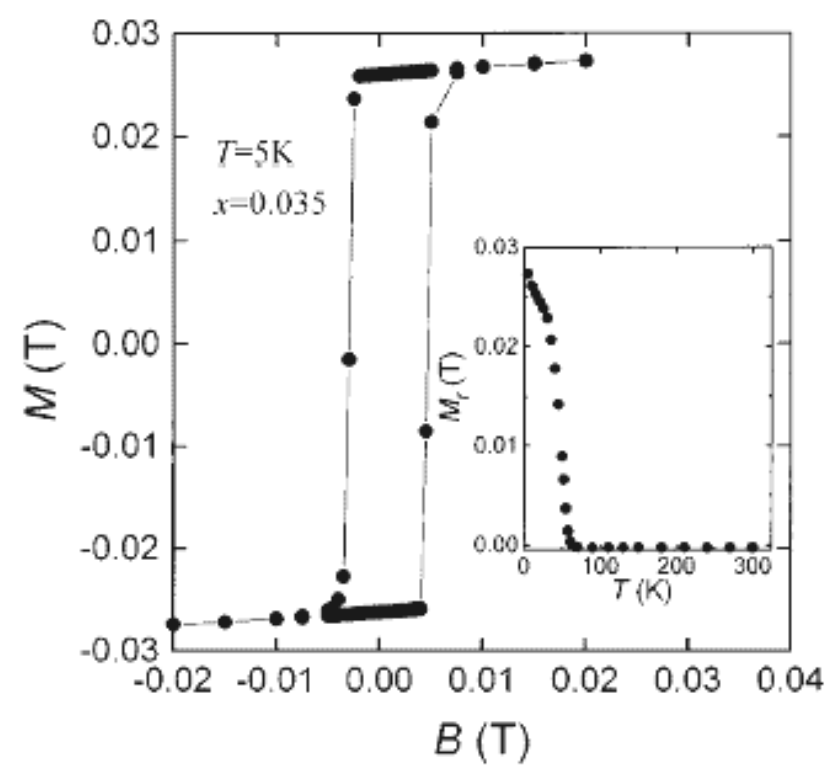

Fig. 8 Magnetic field dependence of magnetization at $5 \mathrm{~K}$ for for (Ga,Mn)As film with Mn $=0.035$. The field was applied parallel to the surface. The inset shows the temperature dependence of remanence of the sample [33]

The origin of increase in remanence below $30 \mathrm{~K}$ as seen in the temperature dependence of remanence (inset fig-8) is not understood. But disappearance of remanence above $75 \mathrm{~K}$ confirmed that MnAs was not the source of ferromagnetic ordering as its $\mathrm{Tc}$ is $310 \mathrm{~K}$. The magnetotransport measurements revealed the occurrence of the anomalous hall effect in the (Ga, Mn)As layer and a carrier concentration was found to be of the order of $\mathrm{Mn}$ concentration. A p-type conductivity was observed.

In another paper Nojiri et al. [65]—reported results of studies of electronic states in $\mathrm{Ga}_{1-\mathrm{x}} \mathrm{M}_{\mathrm{x}} \mathrm{As}$ by using submillimeter ESR. The g-value for these samples was calculated to be $\mathrm{g}$ $=2$ from the frequency dependence. The estimated magnetization from the experiment using this $g$-value was half of the expected value when all $\mathrm{Mn}$ ions take $\mathrm{s}=5 / 2$. The proposed reason was phase separation of $\mathrm{Mn}$ ions or could be that the ferromagnetism of $\mathrm{Ga}_{1-\mathrm{x}} \mathrm{Mn}_{\mathrm{x}} \mathrm{As}$ is itinerant in character, which reduces the magnetization as expected for localized system. The sample with higher Mn concentration had a higher Tc. Still no clear idea for the reason for reduction in magnetisation could be formed.

Typical MBE growth of (Ga, Mn)As is carried out by using solid source MBE with elemental sources $\mathrm{Ga}, \mathrm{Mn}$, and As, usually without intentional doping. Mn provides both localized spins and holes due to its acceptor nature. Epitaxial films of (Ga, Mn)As are grown on semi-insulating GaAs (001) substrates at a typical growth rate of $0.6-0.8 \mu \mathrm{m} /$ hour by De Boek et al. [58] under As-stabilized conditions. Normally, either a GaAs buffer layer or an 
(Al,Ga)As buffer layer is grown before epitaxy of (Ga,Mn)As. The Mn composition $x$ in the $\mathrm{Ga}_{1-\mathrm{x}} \mathrm{Mn}_{\mathrm{x}} \mathrm{As}$ films could be determined from measurements of lattice constant, $a_{0}$, by X-ray diffraction (XRD), once the dependence $a_{\mathrm{o}}$ on $x$ is calibrated by other means, such as electron probe micro-analysis (EPMA).

Properties of $(\mathrm{Ga}, \mathrm{Mn})$ As films do depend on the growth parameters such as arsenic overpressure and substrate temperature, $T_{\mathrm{S}}[66,67]$. However, as long as the established growth procedure is followed, the properties of $(\mathrm{Ga}, \mathrm{Mn}) \mathrm{As}$ films are reproducible; for example, for a given $\mathrm{Mn}$ composition $x$, the ferromagnetic transition temperature $T_{\mathrm{C}}$ can always be maintained in the range of $2000 x \pm 10 \mathrm{~K}$ [68].

Reflection high-energy electron diffraction (RHEED) patterns are used to monitor the surface reconstruction during the growth of films. If the Mn flux or substrate temperature, $\mathrm{T}_{\mathrm{S}}$, is too high, a secondary phase of MnAs is formed. A too low $T_{\mathrm{S}}$ results in a polycrystalline material. The maximum value of $\mathrm{x}$ obtained so far is about 0.07 at $250^{\circ} \mathrm{C}$, and increases up to $\sim 0.10$ at $T_{\mathrm{S}}=200^{\circ} \mathrm{C}[69]$. Attempts to increase $\mathrm{x}$ even further have so far been unsuccessful because of the surface segregation that occurs even at low growth temperatures. Shen et al. show that, at a fixed value of $x=0.035$, epitaxial (Ga,Mn)As films can be grown at $T_{\mathrm{S}}$ varying from 160 to $320^{\circ} \mathrm{C}[70]$.

The homogeneity of as-grown $(\mathrm{Ga}, \mathrm{Mn}) \mathrm{As}$ and the precipitation of MnAs clusters after annealing at around $600^{\circ} \mathrm{C}$ are confirmed by transmission electron microscopy (TEM) [58].The disappearance of the ( $\mathrm{Ga}, \mathrm{Mn})$ As phase by annealing at temperatures above $400^{\circ} \mathrm{C}$ is also confirmed by the disappearance of X-ray diffraction peaks of (Ga,Mn)As [70]. Lowtemperature annealing at $\sim 300^{\circ} \mathrm{C}$ changes lattice constant, magnetic properties, and electrical properties of $(\mathrm{Ga}, \mathrm{Mn}) \mathrm{As}$, which is thought to result from the evaporation of As atoms that form complexes with Mn acceptors [71].

Ferromagnetism in MBE grown (Ga, Mn)As on GaAs (411)A and Si (001) has also been reported by Zhao et al. and Slupinski et al. [72,73] .

\subsection{2 (Ga,Mn)P}

The use of $\mathrm{GaP}$ as a host semiconductor for magnetic dopants is advantageous as it has a large band gap of $2.2 \mathrm{eV}$. Also it is an important component of the AlGaInP materials system which are used for visible LEDs and high speed electronics, and is also nearly lattice matched to Si circuitry [74].

Ferromagnetism above room temperature in $(\mathrm{Ga}, \mathrm{Mn}) \mathrm{P}$ has been reported for two different methods of $\mathrm{Mn}$ incorporation, namely, ion implantation [74,75] and doping during 
MBE growth [76]. The implantation process is efficient for rapidly screening whether particular combinations of magnetic dopants and host semiconductors are promising in terms of ferromagnetic properties.

The temperature-dependent magnetization of a strongly $p$-type $\left(p \sim 10^{20}\right)$, carbon-doped GaP sample implanted with $\sim 6$ at \% of $\mathrm{Mn}$ and then annealed at $700{ }^{\circ} \mathrm{C}$, is shown in Fig. 9(a) [74]. The diamagnetic contribution was subtracted from the background. A Curie temperature $\left(T_{C}\right)$ of $270 \mathrm{~K}$ is indicated by the dashed vertical line, while the inset shows a ferromagnetic Curie temperature of $236 \mathrm{~K}$. Examples of hysteresis loops from the same samples are shown in Fig. 9(b). The hysteresis could be detected to $330 \mathrm{~K}$.
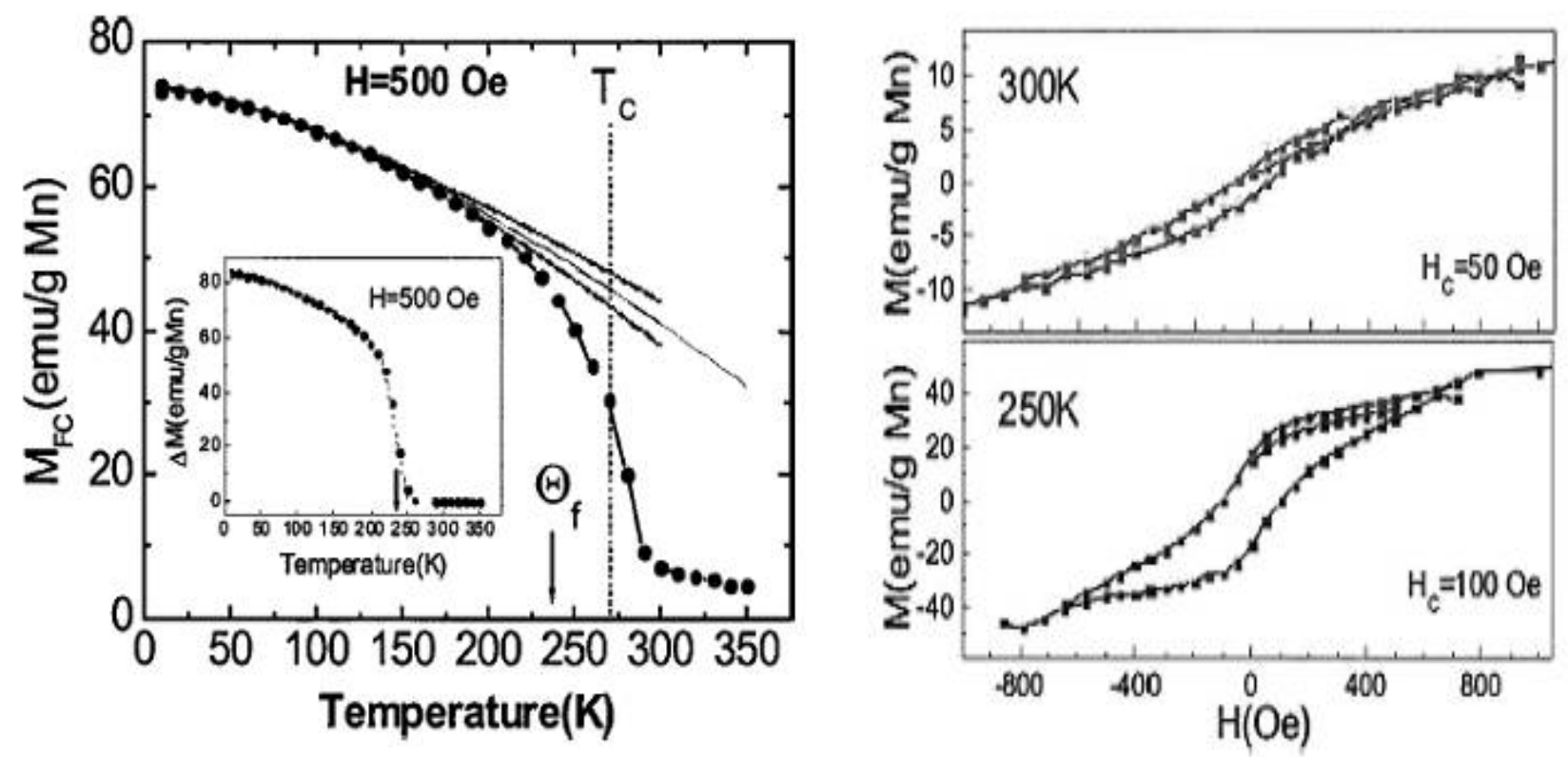

Fig.9 (a) Field cooled magnetization of ( $G a, M n) P$ as a function of the temperature. Solid line shows the bloch law dependence while the dashed lines are 95\% confidence bands. The inset shows the temperature dependence of the difference in magnetization between field cooled and zero filed cooled conditions. (b) Magnetization loops for (Ga, Mn) P at $300 K$ top, and $250 \mathrm{~K}$ (bottom) [74]

When Mn was implanted under the same conditions into $n$-GaP, the $T_{C}$ was reduced to $\sim 50 \mathrm{~K}$. No secondary phases, such as $\mathrm{MnGa}$ or $\mathrm{MnP}$ or clusters were determined by transmission electron microscopy, X-ray diffraction or selected-area diffraction pattern analysis. Mn was incorporated during $\mathrm{MBE}$ growth of the $(\mathrm{Ga}, \mathrm{Mn}) \mathrm{P}$. The $p$-type doping level was separately controlled by incorporating carbon from a $\mathrm{CBr}_{4}$ source, while $\mathrm{P}$ is obtained from thermal cracking of $\mathrm{PH}_{3}$, hence it was possible to tailor the magnetic properties of the n-type $(\mathrm{Ga}$, 
Mn)P. For samples grown at $600{ }^{\circ} \mathrm{C}$ with 9.4 at $\%$ Mn, hysteresis was still detectable at $300 \mathrm{~K}$, with a coercive field of $\sim 39$ Oe as shown in Fig.10 [3].

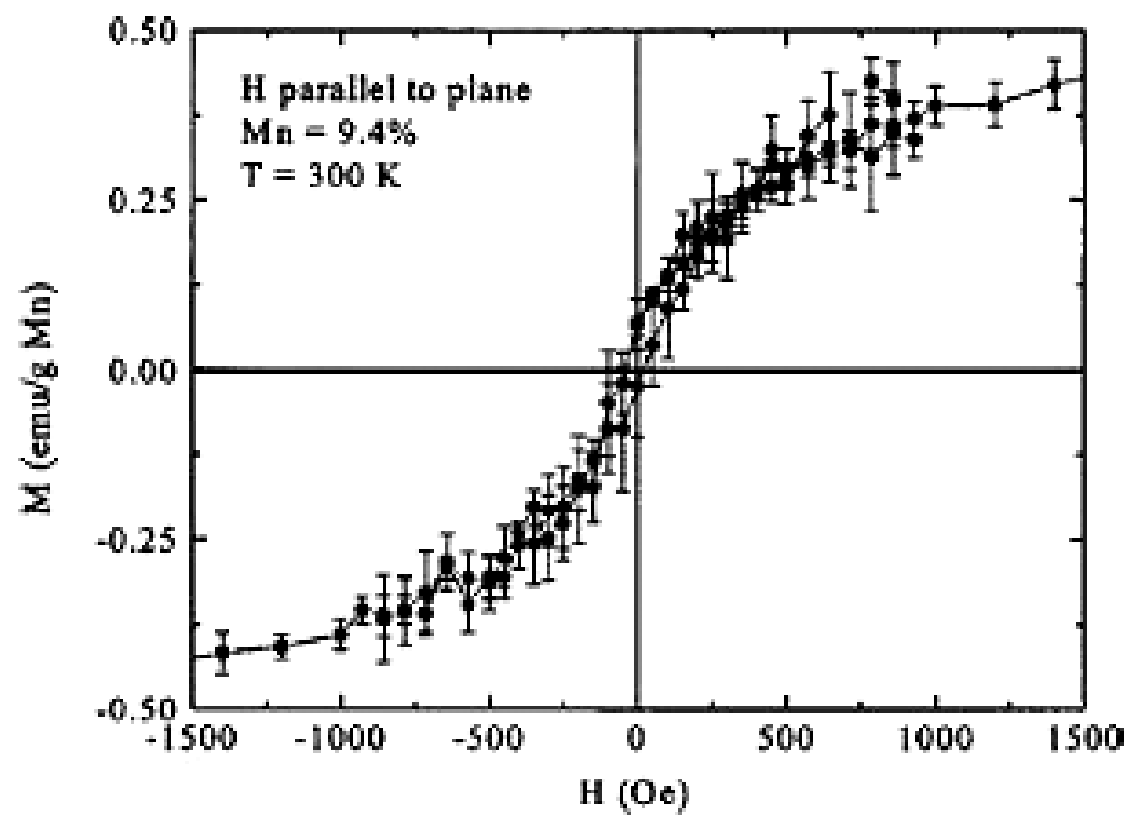

Fig. 10 Magnetization vs field for MBE grown (Ga Mn)P:C with 9.4 at\% Mn during growth [3]

MBE growth of GaP:C with a high concentration of $\mathrm{Mn}$ resulted in the formation of the ferromagnetic phase GaMnP:C. Negative magnetoresistance was observed up to $200 \mathrm{~K}$, while the anomalous Hall effect was observed at 50, 100, and $200 \mathrm{~K}$. No ferromagnetic MnP phase was detected within the film by XRD analysis and cross sectional TEM of the GaMnP:C material in combination with the observed 3D RHEED pattern [76].

\subsection{3 (Ga, Mn)N}

The initial work on this material involved either microcrystals synthesized by nitridization of pure metallic Ga in supercritical ammonia or bulk crystals grown in reactions of $\mathrm{Ga} / \mathrm{Mn}$ alloys on $\mathrm{GaN} / \mathrm{Mn}$ mixtures with ammonia at $1200{ }^{\circ} \mathrm{C}$ [77]. These samples exhibit ferromagnetic properties over a broad range of Mn concentrations, as did some of the other early MBE-grown films. $\mathrm{Ga}_{1-\mathrm{x}}, \mathrm{Mn}_{\mathrm{x}} \mathrm{N}$ bulk crystals with $\mathrm{x}$ up to 0.02 grown by [78] resublimation method and microcrystals with $\mathrm{x}$ up to 0.005 obtained using ammonthermal method by Zajac et al.[77], show extra peaks in the Raman spectra due to disorder coming from $\mathrm{Mn}$ incorporation. Magnetization measurements reveal that these compounds are 
paramagnetic while from the temperature dependence antiferromagnetic nearest-neighbor interaction coupling $(\sim-2 \mathrm{~K})$ is obtained [79]

Structural properties of $(\mathrm{Ga}, \mathrm{Mn}) \mathrm{N}$ with $\mathrm{x}$ up to 0.09 on $\mathrm{AlN}$ buffer onto $\mathrm{Al}_{2} \mathrm{O}_{3}$ substrate grown at a substrate temperature, $T_{\mathrm{S}}=650-750^{\circ} \mathrm{C}$ by $\mathrm{MBE}$ using RF-nitrogen plasma source have been investigated by Kuwabara et al [80,81]. Both cross-sectional TEM and plane-view scanning electron microscopy (SEM) images show no visible second phase. $\mathrm{Mn}$ indeed is incorporated at $\mathrm{Ga}$ sites seen by Extended X-ray absorption fine structure (EXAFS) analysis. Both, ferromagnetic and paramagnetic contributions were present as revealed by magnetic measurements. The origin of ferromagnetism was attributed to ferromagnetic or ferrimagnetic second phases of Ga-Mn and/or Mn-N. The Curie weiss temperature $\theta$ for the paramagnetic part changed from negative to positive with the increase in Mn concentration.

In 2001 Overberg et al. [82] found ferromagnetic ordering in an n-type (Ga,Mn)N film with $\mathrm{x}=0.07$ on $\mathrm{Al}_{2} \mathrm{O}_{3}$ substrate grown by $\mathrm{MBE}$ at $865^{\circ} \mathrm{C}$ using a nitrogen plasma source. They observed negative magnetoresistance below $75 \mathrm{~K}$ and anomalous Hall effect at $10 \mathrm{~K}$. The anomalous component of the Hall resistance disappeared at $25 \mathrm{~K}$, which indicated that the $\mathrm{T}_{\mathrm{c}}$ for this GaMnN material was between 10 and $25 \mathrm{~K}$. No indication of a secondary ferrimagnetic phase $\mathrm{Mn}_{4} \mathrm{~N}$ was observed in the RHEED pattern of the epitaxial surface of the film.

Theoretically predicted, room temperature ferromagnetism in GaMnN was realized experimentally by Sonoda et al. [83]. They reported ferromagnetic ordering in MBE grown $\mathrm{Ga}_{1-\mathrm{x}} \mathrm{Mn}_{\mathrm{x}} \mathrm{N}$ films with $\mathrm{x}=0.06$ and 0.09 . The estimated $\mathrm{T}_{\mathrm{c}}$ from temperature dependence of magnetization was $940 \mathrm{~K}$ (Fig. 7). No Mn-Ga phase, which has a $\mathrm{T}_{\mathrm{c}}$ of $750 \mathrm{~K}$ was found in the films. However, the $940 \mathrm{~K}$ result is now considered to be most likely due to MnGa or $\mathrm{Mn}_{4} \mathrm{~N}$ inclusions and not to single-phase $(\mathrm{Ga}, \mathrm{Mn}) \mathrm{N}$. 


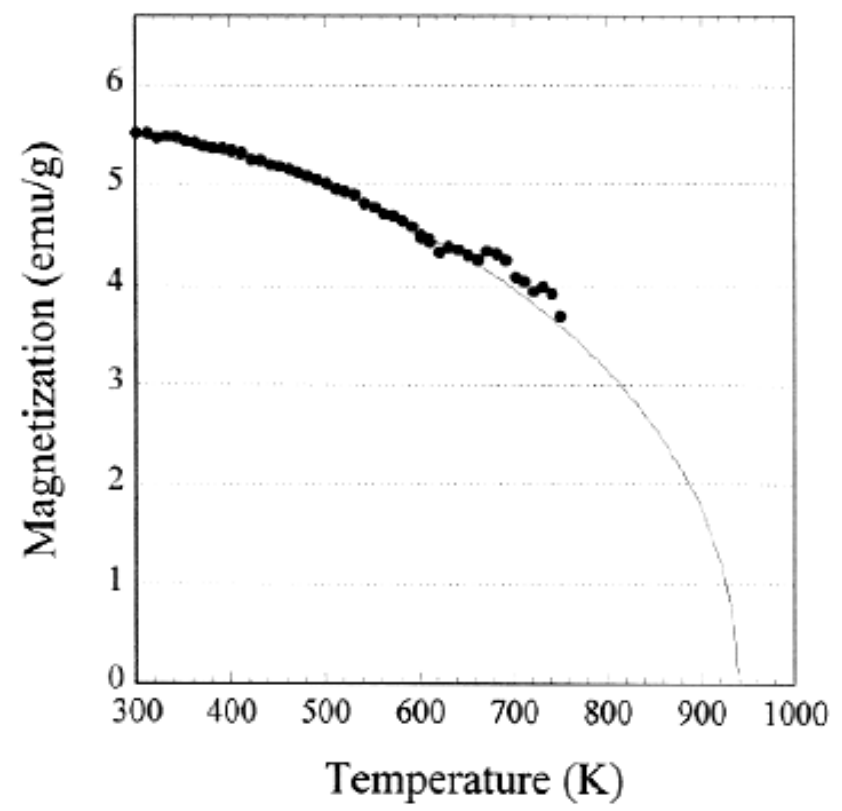

Fig.11 Temperature dependence at 1 KOe for GaMnN film grown by MBE with 9 at\% Mn [83]

The magnetic properties of $\mathrm{p}-\mathrm{GaN}$ implanted with high doses (3 5\%) of $\mathrm{Mn}$ (annealed at $700-1000 \mathrm{oC}$ after doses) have been investigated. The result shows that the sample is ferromagnetic with $T_{\mathrm{C}} \sim 250 \mathrm{~K}$ (Theodoropoulou et al. 2001a). (Ga,Mn)N film prepared by post growth Mn doping using solid state diffusion shows ferromagnetic behavior at room temperature, which is confirmed by the observation of an anomalous Hall effect (Reed et al. 2001). Hence indications of room temperature ferromagnetism have been reported by several groups. Work is under way to rule out the influence of precipitations as well as to establish how $T_{\mathrm{C}}$ depends on the $\mathrm{Mn}$ and carrier concentration.

Besides Mn, Fe was another transition metal which was used for doping GaN. GaN films doped with $\mathrm{Fe}$, with concentrations up to $\sim 3 \times 10^{19} \mathrm{~cm}^{-3}$ were grown by MBE at several substrate temperatures, $T_{\mathrm{S}}$ from 380 to $520^{\circ} \mathrm{C}$ directly on sapphire (0001) substrates. Ferromagnetic behaviour with $T_{\mathrm{C}} \sim 100 \mathrm{~K}$ is observed only in the sample grown at $\sim 400{ }^{\circ} \mathrm{C}$, in spite of quite a low concentration of $\mathrm{Fe}$ [85]. GaN:Fe films (Fe up to $6 \times 1021 \mathrm{~cm}-3$ ) grown by $\mathrm{MBE}$ at substrate temperature of $500 \sim 800^{\circ} \mathrm{C}$ also showed a superparamagnetic behaviour $[80,81]$. 


\subsection{4 (Cd,Mn) GeP2}

Medvedkin et al [26] reported room temperature ferromagnetism in II-VI-V2 chalcopyrite DMS, $\left(\mathrm{Cd}_{1-x} \mathrm{Mn}_{x}\right) \mathrm{GeP}_{2}$ prepared by the solid phase chemical reaction. $\mathrm{Mn}$ vacuum deposition $(30 \mathrm{~nm})$ on a single crystal of $\mathrm{CdGeP}_{2}$ and the reacting process $\left(500^{\circ} \mathrm{C}, 30\right.$ min) was carried out in an MBE Chamber. The average $\mathrm{Mn} / \mathrm{Cd}$ ratio was determined as $20 \%$ for an effective thickness $0.5 \mu \mathrm{m}$ by energy dispersive X-ray analysis. The value $T_{\mathrm{C}} \sim 320 \mathrm{~K}$ of $\left(\mathrm{Cd}_{1-\mathrm{x}} \mathrm{Mn}_{\mathrm{x}}\right) \mathrm{GeP}_{2}$ was determined by magnetization measurements. Clear hysteresis in the field dependence of magnetization and the stripe magnetic domain pattern were observed by magnetic force microscopy (MFM) even at room temperature.

Results of a first principles calculation shows that the antiferromagnetic state is more stable than the ferromagnetic state, and that the energy gap decreases with the $\mathrm{Mn}$ composition [85]. The reason for the discrepancies between theoretical expectations and experimental results is not clear; More recent plane-wave pseudopotential and KKR-CPA calculations shows that the intrinsic defects are responsible for the stabilization of the ferromagnetic $[86,87]$. 


\subsection{Oxide semiconductors}

Compared to non-oxide semiconductors, the oxide semiconductors have many advantages. Their wide band gap makes them transparent and also suitable for applications with short wavelength. They can be easily grown at low temperature even on a plastic substrate and are ecologically safe and durable beside being low in cost. In addition, strong electronegativity of oxygen is expected to produce strong $\mathrm{p}-\mathrm{d}$ exchange coupling between band carriers and localized spins, a prerequisite for DMS [88]. Summarized below are some of the reports on oxide semiconductor based DMS.

\subsection{1 $\mathrm{TiO}_{2}$ based DMS}

$\mathrm{TiO}_{2}$ has been extensively studied for its unique properties, both physical and chemical properties like high refractive index, excellent optical transmittance in the visible and nearinfrared region, and high dielectric constant and photocatalysis for water cleavage. $\mathrm{TiO}_{2}$ exists in three kinds of crystal structure, rutile, anatase, and brookite. They are wide band gap semiconductors. Anatase $\mathrm{TiO}_{2}$ has high mobility of n-type charge carriers $[89,90]$.

It has been shown by Matsumoto et al. [89] that anatase form of $\mathrm{TiO}_{2}$ doped with several percents of $\mathrm{Co}$ are ferromagnetic at room temperature. Films of $\mathrm{Ti}_{1-\mathrm{x}} \mathrm{Co}_{\mathrm{x}} \mathrm{O}_{2}$ with different $x$ values were made on $\mathrm{LaAlO}_{3}(001)$ and $\mathrm{SrTiO}_{3}(001)$ single crystal substrates. No phase segregation was indicated by TEM images. Ferromagnetic long range ordering was seen in anatase $\mathrm{TiO}_{2}$ doped with $\mathrm{Co}$ in the images taken by scanning superconducting quantum interference device (SQUID) microscope. An increase in spontaneous magnetisation with the increase in Co content is observed. Presence of hysteresis in the magnetisation curve indicated room temperature ferromagnetism, with magnetic moment of 0.32 Bohr magnetons per cobalt atom. The films were transparent in the visible and infrared region and had a band gap of $3.1 \mathrm{eV}$. Matsumoto et al. agree that a possibility of Co clusters cannot be completely ruled out though, XRD and TEM showed no sign of metal granules. In a later article Matsumoto et al. state that room temperature ferromagnetism exists in rutile phase of $\mathrm{Ti}_{1-\mathrm{x}}$ $\mathrm{Co}_{\mathrm{x}} \mathrm{O}_{2}$ with a moment of $1 \mu_{\mathrm{B}} / \mathrm{Co}$ atom for $3 \%$ Co substitution.

Chambers et al [91] have grown $\mathrm{Ti}_{1-\mathrm{x}} \mathrm{Co}_{\mathrm{x}} \mathrm{O}_{2}$ films by molecular beam epitaxy on $\mathrm{SrTiO}_{3}$ substrates. They report a magnetic moment per cobalt atom of $1.26 \mu B$ for films with $\mathrm{x}=0.03$, significantly higher than the moment reported by Matsumoto et al. [89]. Chambers 
et al. also report that the cobalt distribution in their films depends strongly on growth conditions leading to some of their films being nonmagnetic. From X-ray absorption spectroscopy measurements, they determine that in the magnetic films the cobalt ions are in the $2^{+}$oxidation state, and substitute on the $\mathrm{Ti}$ site of $\mathrm{TiO}_{2}$. Thus a moment of $1 \mu B / \mathrm{Co}$ is expected. In nonmagnetic films, they found that cobalt had segregated to the surface of the film. In a later report Chambers et al. [92] report segregation of Co-enriched $\mathrm{TiO}_{2}$ anatase clusters nucleate on epitaxial $\mathrm{TiO}_{2}$ anatase grown on $\mathrm{LaAlO}_{3}(001)$ by oxygen plasma assisted MBE. In extreme case almost all the incident Co segregates to the clusters yielding a nanosclae ferromagnetic phase that is not ferromagnetic in homogeneous films of the same Co concentration. Room temperature ferromagnetism (moment $\sim 1.7 \mathrm{uB}$ ) in $\mathrm{TiO}_{2}: \mathrm{Co}$ transparent films grown on both $\mathrm{LaAlO}_{3}(001)$ and $\mathrm{SrTiO}_{3}(001)$ substrates by Pulsed laser deposition (PLD) is reported by Stampe et al also [10]. Cobalt segregation was seen in TEM images. Ferromagnetism originating from Co nanoclusters has been reported by Kim et al. in epitaxial $\mathrm{TiCoO}_{2}$ films [93]. MCD studies done on Co doped anatase $\mathrm{TiO}_{2}$ films also revealed MCD spectral line shape identical to Co metal [94]. In a very recent report Han et al. [90] report ferromagnetism in crystalline $\mathrm{Co}-\mathrm{TiO}_{2}$ grown by co-sputtering, Tc being higher than $400 \mathrm{~K}$. Despite all this work, the key question still remains to be answered is that if Co is indeed substitutional or forms clusters to form ferromagnetic regions within the films.

\subsection{2 $\mathrm{SnO}_{2}$ based DMS}

$\mathrm{SnO}_{2}$ is a n-type semiconductor with a wide band gap of $\sim 3.6 \mathrm{eV}$. It has been used has a transparent conducting electrode in solar cells [95] and gas sensors. This compound has a rutile structure with octrahedral coordibation similar to anatase $\mathrm{TiO}_{2}$. Large n-type carrier concentration upto $10^{21} \mathrm{~cm}^{-3}$ can be achieved by antimony doping. The effective electron mass is quite large $\sim 0.3 \mathrm{~m}_{\mathrm{e}}$. These characteristics are important for strong magnetic exchange interactions in terms of carrier induced magnetism.

Epitaxial films of $\mathrm{Mn}$ doped rutile- $\mathrm{SnO}_{2}$ were fabricated by PLD technique. Systematic changes in lattice constants, and in-gap absorption is found. However, magnetic measurements revealed almost paramagnetic behavior and no ferromagnetic ordering. $\mathrm{Sb}$ doping resulted in a giant positive magnetoresistance as large as $60 \%$ at $5 \mathrm{~K}$ in $\mathrm{Sn}_{0.95} \mathrm{Mn}$ ${ }_{0.05} \mathrm{O}_{2}: \mathrm{Sb}$ films [95]. There have been few reports on $\mathrm{Mn}$ doped $\mathrm{SnO}_{2}$ so far. However, Ogale et al. [52] achieved high temperature ferromagnetism with a giant magnetic moment in Co-

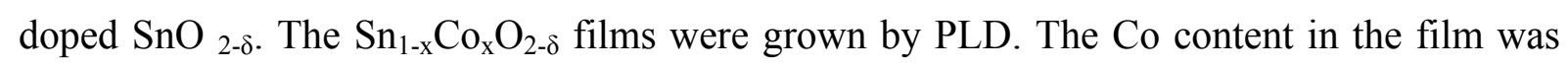


higher than in the target used for ablation due to Sn evaporation. Co concentration was determined by Rutherford back scattering ( RBS). STEM image for a film even with Co content as high as $27 \%(\mathrm{x}=0.27)$ showed a uniform microstructure. EELS data showed uniform distribution of $\mathrm{Co}$ in the film. The magnetization measurement for $\mathrm{Sn}_{1-\mathrm{x}} \mathrm{Co}_{\mathrm{x}} \mathrm{O}_{2-\delta}$ films ( $\mathrm{x}$ from 0.05 to 0.27 ) showed hysteresis at room temperature. A giant magnetic moment of $\sim 7.5 \mu_{\mathrm{B}}$ was found for the film with 5 at $\%$ Co and decreased with increase in Co concentration. A $\mathrm{Tc}$ of $\sim 650 \mathrm{~K}$ was estimated from the temperature dependence of magnetization. Resistivity of films increases with the increase in dopant concentration. The occurrence of giant magnetic moment suggests appearance of moment on the neighbours of cobalt in the matrix, or that, cobalt orbital moment remained unquenched. The origin of giant magnetic moment is not understood properly though, but the results seem promising to explore wide band gap semiconductor $\mathrm{SnO}_{2}$ based DMS.

\subsubsection{Co doped ZnO}

$\mathrm{ZnO}$ based DMS have high potential for functionalities utilizing wide band $(3.3 \mathrm{eV})$ gap, and the large exciton energy $(60 \mathrm{meV})$ [96,97]. It is a well-known piezoelectric and electro-optic material, and can be easily deposited in thin film form. It has wide applications in electronic devices such as transparent conductor, thin film gas sensor, varistor, surface acoustic wave (SAW) devices, optical wave-guides, acousto-optic modulators/deflectors, ultraviolet LASER source, and ultraviolet detectors [13]. It is an n-type semiconductor with the room temperature hall mobility in $\mathrm{ZnO}$ single crystals of the order of $200 \mathrm{~cm}^{2} \mathrm{~V}^{-1} \mathrm{~s}^{-1}$. Eelectron doping via defects originates from $\mathrm{Zn}$ interstitials in the $\mathrm{ZnO}$ lattice. The intrinsic defect levels that lead to $n$-type doping lie approximately $0.05 \mathrm{eV}$ below the conduction band.

There is also interest in developing the use of ion implantation of $\mathrm{ZnO}$ for device doping, and isolation, as well as investigating the effectiveness of different transition metals for magnetic doping. Jin et al. [98] fabricated epitaxial thin films of $\mathrm{ZnO}$ doped with all the $3 \mathrm{~d}$ transition metals by using a Combinatorial laser MBE technique, and found that the solubility of TM ions in $\mathrm{ZnO}$ depend upon their ionic radius and valence sates. According to their findings $\mathrm{M}^{2+}$ and $\mathrm{Co}^{2+}$ ions had very high solubility limits among all the $3 \mathrm{~d}$ ion species, and have a high spin electron configurations in the $\mathrm{ZnO}$ matrix.

$\mathrm{Zn}_{1-\mathrm{x}} \mathrm{M}_{\mathrm{x}} \mathrm{O}$, with $(\mathrm{TM}=\mathrm{Sc}, \mathrm{Ti}, \mathrm{V}, \mathrm{Cr}, \mathrm{Mn}, \mathrm{Co}, \mathrm{Ni}$ and $\mathrm{Cu})$, has been investigated following theoretical prediction of a possibility of ferromagnetic ordering in these compounds by [99]. Their ab initio calculations based on local density approximation suggested that FM 
ordering of $\mathrm{Mn}$ is favored when mediated by hole doping. However, for $\mathrm{V}, \mathrm{Cr}, \mathrm{Fe}, \mathrm{Co}$, and $\mathrm{Ni}$ dopants, FM ordering in $\mathrm{ZnO}$ is predicted to occur without the need of additional charge carriers. However, Ando et al. [100] found strong sp-d exchange interactions in $\mathrm{Mn}, \mathrm{Co}$, Ni and $\mathrm{Cu}$ doped $\mathrm{ZnO}$, whereas in $\mathrm{Sc}, \mathrm{Ti}, \mathrm{V}$ and $\mathrm{Cr}$ doped samples these interactions were absent. Ueda et al. [36] also reported maximum solubility of $\mathrm{Co}$ in $\mathrm{ZnO}$ as compared to other transition metal dopants. Ferromagnetic behaviour with a Curie temperature above room temperature was observed only in Co doped ZnO films deposited by PLD. The mechanism of ferromagnetism was not clear, though. Another group in Korea, Yoon et al., [101] also studied magnetic properties of TM doped ZnO based DMS. They focused their studies on the powder and bulk samples and found antiferromagnetic interactions between transition metal ions. However there are reports of room temperature ferromagnetism in $\mathrm{Zn}_{1-\mathrm{x}} \mathrm{TM}_{\mathrm{x}} \mathrm{O}$ (TM= $\mathrm{V}, \mathrm{Fe}, \mathrm{Ni}$ ) besides $\mathrm{Zn}_{1-\mathrm{x}} \mathrm{Co}_{\mathrm{x}} \mathrm{O}$ and $\mathrm{Zn}_{1-\mathrm{x}} \mathrm{Mn}_{\mathrm{x}} \mathrm{O}$ (table-1). Since most of the positive results were found with $\mathrm{Co}$ doped $\mathrm{ZnO}$, therefore an intensive research is being carried out on Co doped $\mathrm{ZnO}$ DMS.

Ando et al. [102] observed strong magneto-optical effect of $\mathrm{Zn}_{1-\mathrm{x}} \mathrm{Co}_{\mathrm{x}} \mathrm{O}$ near the optical band gap. Magnetic circular dichorism (MCD) and Faraday rotation for $(\mathrm{x}=0.012$ and 0.016$)$ at $5 \mathrm{~K}$ was two orders higher than for $\mathrm{ZnO}$. These magnetooptical effects disappear at room temperature. Though Ueda et al. [36] had reported ferromagnetism in Co doped $\mathrm{ZnO}$ films but reproducibility was less than $10 \%$. Jin et al. didn't find any ferromagnetic ordering at all in their films grown by laser MBE [98]. Cho et al. reported ferromagnetism in CoFe doped $\mathrm{ZnO}$ films grown by reactive magnetron cosputtering [103]. The solubility limit of CoFe ions in $\mathrm{ZnO}$ was determined to be more than $15 \%$. The origin of ferromagnetism is still to be clarified. Following this, Lee et al [104] reported a Tc above $350 \mathrm{~K}$ in Co doped ZnO films fabricated by sol-gel method grown on (0001) $\mathrm{Al}_{2} \mathrm{O}_{3}$ substrate. No additional doping was done and the films samples were insulating. Secondary phases were formed on increasing the Co concentration above 25\%. Similar results were obtained by Prellier et al. [105]. Their $\mathrm{Co}: \mathrm{ZnO}$ films deposited by laser ablation using Co and $\mathrm{Zn}$ metal targets in an oxidizing atmosphere exhibited near room temperature ferromagnetism when Co concentration was 5\%. Whereas, Risbud et al. [106] found no ferromagnetism in $\mathrm{Zn}_{1-\mathrm{x}} \mathrm{Co}_{\mathrm{x}} \mathrm{O}$ with Co concentration of 5,10 and $15 \%$.

Hence different research groups reported different results. This controversy between research teams may result from difference in synthesis techniques or the growth conditions used for making films. A clear picture regarding mechanism of ferromagnetism in Co doped $\mathrm{ZnO}$ has not emerged yet. Co clusters or $\mathrm{CoO}$ grains might be the sources of ferromagnetism. 


\section{7. $\mathrm{Mn}$ doped $\mathrm{ZnO}$}

The wonder material!

According to the theoretical prediction, FM ordering in TM doped $\mathrm{ZnO}$ can be achieved without using any additional carriers with $\mathrm{V}, \mathrm{Cr}, \mathrm{Fe}, \mathrm{Co}$ and $\mathrm{Ni}$ [99] whereas with Mn doping additional p-type doping is needed, [99,9]. Despite this Mn remains the primary dopant of interest because solubility of $\mathrm{Mn}$ in $\mathrm{ZnO}$ is larger than $10 \mathrm{~mol} \%$ and the electron mass is as large as $\sim 0.3 m_{e}$, where $m_{e}$ is the free electron mass [107]. Infact, Fukumura et al. showed that $\mathrm{Mn}$ atoms could be doped into $\mathrm{ZnO}$ upto $35 \%$ by PLD technique without phase segregation [96]. Therefore, the amount of injected spin in the host material can be very large with Mn doping. Moreover, $\mathrm{Mn}$ is known to be anitiferromagnetic, which makes this system more clean in terms of metal precipitate induced ferromagnetism, which is the subject of great controversies in DMS (eg in Co doped $\mathrm{TiO}_{2}$, Co doped $\mathrm{ZnO}$ )

The model by Dietl et al predicts that the transition temperature in dilute magnetic semiconductors (DMS) will scale with a reduction in the atomic mass of the constituent elements due to an increase in $p-d$ hybridization and a reduction in spin-orbit coupling. The theory predicts a $T_{\mathrm{C}}$ greater than $300 \mathrm{~K}$ for $p$-type $\mathrm{ZnO}$ doped with $\mathrm{Mn}$ mediated by heavy $\mathrm{p}$ type doping, with $T_{\mathrm{C}}$ dependent on the concentration of magnetic ions and holes [9].

This prediction by Dietl initiated persuit of a Mn doped ZnO based DMS. However, no reports for confirmation of the theoretical work appeared until 2003 [53]. Fukumura et al. found a spin-glass behaviour [96]. Tiwari et al.did not find any ferromagnetism [97] in their $\mathrm{Zn}_{1-\mathrm{x}} \mathrm{Mn}_{\mathrm{x}} \mathrm{O}$ films deposited on sapphire substrate using PLD. Jung et al. [108] reported that ferromagnetism appears at low temperature in $\mathrm{Zn}{ }_{1-\mathrm{x}} \mathrm{Mn}_{\mathrm{x}} \mathrm{O}$ films grown on $\mathrm{Al}_{2} \mathrm{O}_{3}(001)$ substrates using laser MBE. The transition temperature obtained from magnetization measurements was $30 \mathrm{~K}$ for $\mathrm{x}=0.1$ film and $45 \mathrm{~K}$ for $\mathrm{x}=0.3$ one. Similar results were reported by Han et al. [109] in Mn doped bulk sampled prepared by solid state reaction at processing temperature of $1170 \mathrm{~K}$ and $1370 \mathrm{~K}$ with $\mathrm{Mn}$ concentration of $5 \mathrm{at} \%$. The former sample showed a ferromagnetic transition at $30 \mathrm{~K}$, whereas the sample processed at higher temperature was paramagnetic down to $4 \mathrm{~K}$. The $\mathrm{T}_{\mathrm{C}}$ of $30 \mathrm{~K}$ seen in $1170 \mathrm{~K}$ processed sample was attributed to a secondary phase $(\mathrm{Mn}, \mathrm{Zn}) \mathrm{Mn}_{2} \mathrm{O}_{4}$ which is known to have a ferrimagnetic transition at low temperatures.

In all of these studies the $\mathrm{ZnO}$ material was n-type. Little is known about ferromagnetism in p-type $\mathrm{Zn}{ }_{1-\mathrm{x}} \mathrm{Mn}_{\mathrm{x}} \mathrm{O}$ films due to difficulty in synthesizing p-type $\mathrm{ZnO}$ 
films. Joseph et al. have reported on successful preparation of p-type $\mathrm{ZnO}$ by doping with nitrogen as an acceptor element but there still exists some obstacles such as poor reproducibility [110].

The confirmation to the theory by Dietl et al. came with the the report by Parmanand et al. [13] on the observation of room temperature ferromagnetism in Mn doped $\mathrm{ZnO}$. Despite the uncertainty in the mechanism of ferromagnetism in the system and the fact that the obtained magnetisation is much lower than the theoretically predicted value of $5 \mu_{\mathrm{B}}$ per Mn ion, these results provide a pathway for exploring it further. Following this discovery, Lim et al. also report on hole mediated ferromagnetism in $\mathrm{Zn}_{1-\mathrm{x}} \mathrm{Mn}_{\mathrm{x}} \mathrm{O}$ thin films $(\mathrm{x}=0.03$ and 0.20$)$ prepared on GaAs substrate by the rf magnetron cosputtering method [111]. At a substrate temperature high enough, As from the substrate could diffuse into the film and p-type films were synthesized. Even though the origin of ferromagnetism in the films is not clear the magnetic measurements showed a clear room temperature ferromagnetism in p-type $\mathrm{Zn}_{1 \text { - }}$ ${ }_{\mathrm{x}} \mathrm{Mn}_{\mathrm{x}} \mathrm{O}$ films.

According to Dietl et al [9], Ferromagnetism in Mn based DMS can be achieved provided that the hole density is sufficiently high. According to this theory the Mn ions substituted at $\mathrm{Zn}$ site provide the local spin. The ferromagnetic correlations are mediated by holes from shallow acceptors in a matrix of these localized Mn spins. These holes mediate ferromagnetic interactions between nearest $\mathrm{Mn}-\mathrm{Mn}$ sites which are otherwise antiferromagnetically coupled. The hole doping can be accomplished by adding an acceptor impurity. Jun et al. [112] showed that adding $\mathrm{Cu}$ to n-type $\mathrm{ZnO}$ increases resistivity to upto $1 \%$ mixture which showed that $\mathrm{Cu}$ can be a p-type dopant. Following this, room temperature ferromagnetism with $\mathrm{T}_{\mathrm{c}}$ as high as $500 \mathrm{~K}$, in $\mathrm{Cu}$ doped $\mathrm{Zn}$ 1-x $_{\mathrm{x}} \mathrm{Fe}_{\mathrm{x}} \mathrm{O}$ was achieved [113]. Computational studies using density functional theory (DFT) approach based on pseudopotentials with localised atomic-orbital basis sets, N. A. Spladin found that for pure $\mathrm{Mn}$ doped $\mathrm{ZnO}$ the energy difference between ferromagnetic, and antiferromagnetic alignment is small (of the order of $\mathrm{meV}$ ) with the antiferromagnetic state is more favorable for separated Mn configuration, and ferromagnetic state being more favorable for close $\mathrm{Mn}$ configuration [114]. The energy difference in ferro and antiferromagnetic state, with ferromagnetic state stable was found to increase with the additional doping of $\mathrm{Cu}$ in $\mathrm{Mn}$ doped $\mathrm{ZnO}$. The additional dopping of $\mathrm{Cu}$ in $\mathrm{Mn}$ dopped $\mathrm{ZnO}$ is used to provide holes to mediate the ferromagnetic coupling between $\mathrm{Mn}-\mathrm{Mn}$ in $\mathrm{Mn}$ doped $\mathrm{ZnO}$.

In case of electron doped Mn based DMS, ferromagnetism either doesn't occur at all or occurs at very low temperature. However, Norton et al. observed ferromagnetic ordering in 
n-type Mn-implanted, Sn-doped $\mathrm{ZnO}$ crystals, [115] where $\mathrm{Sn}$ is a doubly ionized donor impurity. The Curie temperature is quite high, approaching $250 \mathrm{~K}$. The Sn may simply provide carriers, albeit electrons, which effectively mediate the spin interactions or which could alternatively form complexes with $\mathrm{Mn}$, resulting in both $\mathrm{Mn}^{+2}$ and $\mathrm{Mn}^{+3}$ sites that could yield a ferrimagnetic ordering. However, codoping with Al resulted in n-type material with carrier concentration in excess of $10^{19} \mathrm{~cm}^{-3}$. Large magnetoresistance was observed in the films but no ferromagnetism was reported [115].

In view of these findings one can say that carrier concentration is not the only parameter that influences the magnetic properties of $\mathrm{Mn}: \mathrm{ZnO}$. This is consistent with work by Theodoropoulou et al. who found that FM in $\mathrm{ZnO}$ films deposited by reactive magnetron sputtering was strongly dependent on parameters such as growth temperature, $\mathrm{O}_{2}$ partial pressure, and type of substrate (only films deposited on $\mathrm{Al}_{2} \mathrm{O}_{3}$ substrates were FM). Nonoptimized growth conditions produced weakly paramagnetic behaviour [116]. 


\section{Experimental Work, and Characterization Techniques:}

\subsection{Sample preparation : Solid -Solid Reaction Method}

The samples were prepared by standard solid solid reaction. Appropriate amount of precursors $\mathrm{ZnO}(99.99 \%)$ and $\mathrm{MnO}_{2}$ (99.99\%) were mixed and ground properly in order to obtain sub-micron size precursors. The mixture was then calcined at $400^{\circ} \mathrm{C}$ for $8 \mathrm{hrs}$ in air or appropriate atmoshpere of Oxygen, Nitrogen, or hydrogen. The calcined powder thus obtained was pressed to form dense pellets and sintered for $12 \mathrm{hrs}$ at temperatures ranging from $500^{\circ} \mathrm{C}$ to $900^{\circ} \mathrm{C}$ in suitable atmospheres mentioned above. In the case of Co doping with $\mathrm{Cu}, \mathrm{CuO}$ was also ground thoroughly with $\mathrm{MnO}_{2}$ and $\mathrm{ZnO}$ before calcination.

The pellets thus obtained were characterized for physical as well as magnetic properties and ablated by Pulsed laser ablation to make thin films

\subsection{Techniques}

In the experiments carried out in this thesis we have used the following Characterization techniques:

1) Pulsed Laser Ablation

2) X-ray Diffraction

3) Soft X-ray spectroscopies: (at the Advanced Light Source Division, Berkeley National Laboratories in collaboration with Dr. Jinghua Guo)

> X-ray absorption, XAS,

$>$ X-ray emission, XES, and

$>$ Resonant inelastic X-ray scattering, RIXS spectroscopies

4) Scanning electron microscopy and Energy dispersive spectroscopy

5) Transmission electron microscopy

6) Selected area electron diffraction

7) Electron energy loss spectroscopy

8) SQUID magnetometry

9) Ferromagnetic resonance spectroscopy 
The pulsed laser Ablation (PLA) technique is the most suitable one for deposting thin films in a laboratory [117]. In laser ablation, high-power laser pulses are used to evaporate matter from a target surface such that the stoichiometry of the material is preserved in the interaction. As a result, a supersonic jet of particles (plume) is ejected normal to the target surface. The plume, expands away from the target with a strong forward-directed velocity distribution of the different particles. The ablated species condense on the substrate placed opposite to the target. The ablation process takes place in a vacuum chamber - either in vacuum or in the presence of some background gas.

PLA has shown its unique advantage of depositing complex oxide compounds. It is relatively easier to replicate the stoichiometry of the target composition in the deposited films, convenient to oxygenate films in-situ by reactive deposition in relatively high oxygen pressure, flexible to deposit multilayers, and above all simple operation procedures and so on. The targets for PLA are relatively small, usually not greater than $25 \mathrm{~mm}$ in diameter and therefore relatively inexpensive and allows studies on composition variation to be readily performed . An additional advantage of PLA in the deposition of thin films is that the films are relatively pinhole free. The kinds of films deposited by PLA expand from high Tc superconductors to piezoelectric, ferroelectric films, and semiconducting oxides.

A typical PLA system, consists of a pulsed laser, a vacuum chamber, a rotating target holder and a substrate heating block. There are several kinds of lasers, which are commercially available, and the choice of laser is on Excimer lasers $(\mathrm{XeCl}, \mathrm{KrF}, \mathrm{ArF})$ are widely used to deposit high Tc superconducting films, and other complex oxide films because of the larger absorption coefficient and small reflectivity of materials at their operating wavelengths. Frequency tripled Nd:YAG lasers are also effective from the same point of view.

Continuum (model NY81-C) Nd:YAG laser operating ( $\lambda=355 \mathrm{~nm}$ ) with a pulse repetition rate of $10 \mathrm{~Hz}$ and an energy density of $1.0 \mathrm{~J} \mathrm{~cm}-2$ was used for the present work.

\subsubsection{X-ray diffraction}

It is a versatile, non-destructive analytical technique for identification and quantitative determination of the various crystalline forms known as phases of compounds present in the powdered and solid samples [118]. Indentification is achieved by comparing the X-ray 
diffraction pattern obtained from unknown sample with an internationally recognized database containing reference patterns for more than 70,000 phases.

A crystal lattice is a regular array of atoms in space. These are arranged in space to form a series of parallel planes separated from each other by distance d, which varies according to the nature of materials. For any crystal planes oriented in different direction has different d spacing.

When a monochromatic X-ray beam with wavelength $\lambda$ is incident on the lattice planes in the crystal at an angle, $\theta$, diffraction occurs only when the distance travelled by rays reflected from successive phases differs by a complete number ' $n$ ' of $\lambda s$. That is, the Bragg's condition given by [118]

$n \lambda=2 d \sin \theta$

By varying $\theta$, the Bragg's law can be satisfied by different ' $d$ ' spacing in a polycrystalline material. Plotting angle position and intensity of the resultant diffraction peaks produces a pattern, which is characteristic of the sample. For a sample containing a mixture of phases the $\mathrm{XRD}$ pattern is formed by addition of individual patterns.

The three basic components of an X-ray diffractometer are: $\mathrm{x}$ - ray source, specimen, and $\mathrm{x}$ - ray detector. They all lie on the circumference of a circle, which is known as the focusing circle. The angle between the plane of the specimen and the X-ray source is $\theta$, the Bragg angle. The angle between the projection of the X-ray source and the detector is $2 \theta$. For this reason the X-ray diffraction patterns produced with such a geometry are often known as $\theta-2 \theta$ scan.

A Philips PW 1830 X-ray diffractometer (XRD) was used in the present work to verify the single phase character of our samples. The shift in peak angles with the addition of dopant in the host and also with the change in dopant concentration gives valuable information about dopant atoms being substitutional in the lattice. Philips X'Pert software was used to obtain precise value of parameters such as peak position, peak counts, $d$ spacing etc. 


\subsubsection{Soft Xray spectroscopies.}

Spectroscopic measurements are powerful tools in modern physics, and the interaction between light and matter is one of the primary experimental areas for probing the properties of solids. Especially important for studying electronic properties is light in the range of soft $\mathrm{x}$ rays and the vacuum ultraviolet, which interacts with electrons in solids, with strength that varies with the photon energy. Such techniques as X-ray absorption spectroscopy, are now standard tools for studying the electronic structure of materials. The application of these techniques and the interpretation of the results are especially important in the studies of exciting new materials, including strongly correlated electron systems, new superconductors, organic materials, and complex systems.

Synchrotron radiation is the light emitted by electrons as they circulate around a high- energy accelerator. The emitted photons in the energy range at or below $1 \mathrm{keV}$ fall in the soft X-ray region [119]. Soft X-ray spectroscopies answer the question-what do the electrons do when migrating between the atoms? Xray originates from an electronic transition between a localized core state and a valence state. As a core state is involved, elemental selectivity is obtained because the core levels of different elements are well separated in energy, meaning that the involvement of the inner level makes this probe localized to one specific atomic site around which the electronic structure is reflected as a partial density-of-states contribution. The participation of valence electrons gives the method chemical state sensitivity and further, the dipole nature of the transitions gives particular symmetry information. Also, the substantial penetration of soft-X-rays offers true bulk probing [119]. Performance of synchrotron radiation improved a great deal since last decade. The new generation synchrotron radiation sources producing intensive tunable monochromatized X-ray beams have opened up a new possibility of soft X-ray emission spectroscopy (SXES). Also, now it is possible to select energy of excitation which makes it possible to perform resonant excitation, i.e., exciting to particular empty states. The introduction of selectively excited softX-ray emission has opened a new field of study by disclosing many new possibilities of resonant inelastic soft-X-ray scattering (RIXS). 


\subsubsection{X-ray absorption and emission spectroscopy}

Soft-X-ray absorption spectrum provides information about the unoccupied states. In an XAS experiment monochromated photons of certain energy impinge on a sample and are absorbed. If the photon energy is high enough, there exists the possibility of the excitation of core level electrons into unoccupied states of the solid. This is measured indirectly via the decay of the corresponding excitation giving rise to fluorescence light or Auger electrons, whose intensity is proportional to the absorption cross-section hence the intensity of these secondary electrons can be measured as a function of incoming photon energy.

$\mathrm{X}$-ray absorption spectroscopy is a powerful technique to investigate the electronic structure of TM compounds using the local process of $2 p$ to $3 d$ absorption. One of the advantages of TM $2 p$ XAS is that the CI analysis of the multiplet structures can give local electronic structure parameters, such as the multiplet and crystal field strengths, more accurately.

The core vacancy left by the excited electron is filled by an electron from the valence orbital. Hence soft-X-ray emission also gives direct information about the chemical bonding. Whereas, X-ray absorption provides information about the unoccupied electronic states, the emission gives information about the occupied ones.

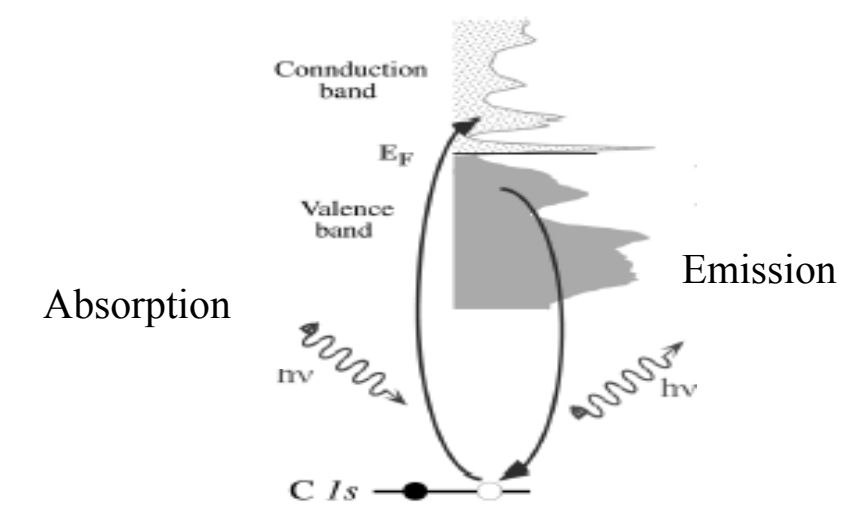

Fig-12 A schematic showing X-ray absorption and emission [119]

\subsubsection{Resonant inelastic soft-X-ray scattering (RIXS)}

If the core electron is resonantly excited to the absorption threshold by the incident photon (as in the process of X-ray absorption spectroscopy), the resulting emission spectrum depends 
strongly on the incident-photon energy $\Omega$, and this type of XES is denoted as resonant X-ray emission spectroscopy (RXES). On the other hand, if the core electron is excited to the highenergy continuum, well above the absorption threshold (as in the process of X-ray photoemission spectroscopy), this type of XES is denoted as normal X-ray emission spectroscopy (NXES), where the spectral shape does not depend on $\Omega$ [120].

Resonant X-ray emission spectra are classified into two categories: when the emitted-photon energy $\omega$ is the same as that of the incident-photon energy $\Omega$, the spectrum is called "'Rayleigh scattering', or "resonant elastic X-ray scattering," while for $\omega ́$ not equal to ' $\Omega$ it is called “'resonant inelastic X-ray scattering (RIXS)'”[120].

RIXS provides us with bulk-sensitive and site-selective information. Furthermore, it is a photon-in and photon-out process, so that this technique is equally applicable to metals and insulators and can be performed in applied electric or magnetic fields, as well as in applied high pressure.

The characteristic features of RIXS depend on materials. For the transition-metal compounds it is interesting to study the interplay between the local and itinerant character of $3 d$ electrons. RIXS for $f$ and $d$ electron systems gives us important information on the electronic states, such as the intraatomic multiplet coupling, electron correlation, and interatomic hybridization [120].

\subsubsection{Scanning Electron microscope (SEM)}

Scanning Electron Microscope (SEM) is becoming a valuable new tool for the nondestructive inspection, examination and evaluation of materials, both metallic and nonmetallic, as well as assemblies and surfaces [121]. The item to be examined and evaluated is placed on a specimen stage inside a vacuum enclosure of the SEM station and is irradiated with a finely focused beam of electrons, with energies typically in the range from a few hundred $\mathrm{eV}$ to about $30 \mathrm{KeV}$, that can be static or swept in a cyclic fashion over the specimen's surface. The resulting signals that are produced when the scanning electron beam impinges on the surface of the specimen include both secondary emission electrons as well as backscattered electrons. These signals vary as the result of differences in the surface topography as the scanning electron beam is swept across the specimen surface.

The secondary emission of electrons from the specimen surface is usually confined to an area near the beam impact zone that permits images to be obtained at a relatively high resolution. These images as seen on a Cathode Ray Tube provide a three dimensional 
appearance due to the large depth of field of the Scanning Electron Microscope (SEM) as well as the shadow relief effect of the secondary electrons contrast. In a typical SEM, a resolution of 100 Angstroms can be attainable, and a depth of field (focus) 300 times that of an optical microscope and having good working distances. The large depth of field available with a SEM makes it possible to observe three-dimensional objects in stereo. The three-dimensional images produced, allow different morphological features to be correctly interrelated and correctly analyzed.

One of the unique advantages of Scanning Electron Microscopy is the fact that many specimens can be examined with minimal specimen preparation activity. The thickness of the specimen is not a consideration. Therefore bulk specimens can be examined in a SEM with a size limited only by the dimensions of the test specimen compared to the dimensions of the SEM's specimen stage within the vacuum enclosure. For the examination and evaluation of a metallic material surface, the only usual amount of specimen preparation is to be sure that the specimen surface to be examined is clean.

A SEM is usually provided with analytical facility. Secondary and backscattered electron images are collected to visualize the surface topography and average chemistry of a specimen respectively. The primary beam electrons displace the inner shell electrons of atoms in a specimen. As a result, those atoms undergo a "relaxation" which results in X-ray radiation being emitted. Because every atom in the periodic table has a unique electronic configuration, the X-rays produced carry a unique atomic signature in the form of a characteristic energy. The EDS method employs a solid state $\mathrm{Si}(\mathrm{Li})$ detector to measure the distribution of X-ray energies, thereby allowing one to identify (and quantify) elemental species. Rastering of the beam across the surface permits one to track the locations of sample X-rays, and hence, 2 dimensional chemical maps can be constructed.

We did elemental analyses for our samples using energy dispersive spectroscopy (EDS: on detector LINK Gem Oxford in a JEOL JSM-840 SEM). The chemical composition and homegeniety of the samples was checked by elemental mapping.

\subsubsection{Transmission electron microscope}

A TEM is a versatile and efficient technique for characterization of materials [121, 122]. The spatial resolution of a microscope depends upon the wavelength of the radiation used. The resolving power of an optical microscope is limited by the wavelength of the light employed: $(\lambda>3000 \AA)$. In the electron microscope, much shorter de Broglie wavelengths are 
possible by using highly energetic electrons, thereby pushing the resolution limit down to the A level. Since they are charged, electron beams can be deflected by electrostatic or magnetic fields. In virtually all commercial electron microscopes nowadays, magnetic lenses are used to focus the electron beam carrying out the functions that glass lenses serve in a conventional optical microscope.

The principle of operation is entirely analogous to the optical microscope. A collimated beam of electrons, emitted from a hot $\mathrm{LaB}_{6}$ filament, is accelerated to $\sim 120 \mathrm{keV}$. After passing through a condenser lens the beam is incident on the sample. The size of the beam can be varied, but is typically $\sim 1$ micron in diameter. Thus small selected areas of the sample can be probed. The beam passes through the sample (which is thinned to $\sim 200 \AA$ to permit transmission of electrons in the $100 \mathrm{KeV}$ range) An objective lens, situated immediately below the sample, then produces a magnified image of the sample. Finally, this image is projected onto a fluorescent screen at the base of the column, which is an electron absorption contrast map in a bright field image

One of the powerful features of electron microscopes is their ability to display diffraction patterns of the sample. In this case the wave-like nature of the electrons is utilized to diffract the incident beam from the atomic structure within the sample. This can provide information on the crystal structure of the sample and is particularly useful when the atomic arrangement is regular and periodic, as in a crystal. It is very straightforward to switch from a real image to the diffraction pattern.

A High Resolution Transmission Electron Microscope can resolve distances smaller than $1 \mathrm{~nm}$ and is used for imaging atomic arrangements in crystals (and quasi-crystals), structural defects interfaces (grain boundaries, interphase interfaces) stacking faults, antiphase boundaries, inversion domain boundaries, crystal dislocations. It is thus a powerful tool for looking at a specimen at atomic scale. It is covenient as the samples don't need to be conductive as in case of STEM or SEM. In case of transition metal doped semiconductor samples it is good technique to verify homogeneous distribution of TM ions in the host matrix without formation of clusters or precipitates which is essential to understand the mechanism of ferromagnetic ordering obtained in these systems.

\subsubsection{Selected Area Electron Diffraction (SAED)}

The charge of an electron makes it to react with both nuclei and electrons in a specimen. In SAED, a selected area is chosen using an aperture. The SAED aperture is placed in an image plane below the specimen and electrons only from a selected area are allowed to contribute to the diffraction pattern. By projecting the back focal plane onto the screen of the 
microscope, an image of the diffraction pattern is obtained. The pattern reveals crystallographic information such as, crystal orientation, interplanar distances, symmetries, and are widely used for crystal structure determination and phase identification.

\subsubsection{Electron energy loss spectroscopy (EELS)}

When electron beam is incident into specimen, a part of the electrons is inelastically scattered and loses a part of the energy. Elemental composition and atomic bonding state can be determined by analyzing the energy with the spectroscope attached under the electron microscope (Electron Energy Loss Spectroscopy). Because the analyzed region can be selected from a part of the enlarged electron microscopic image, one can analyze very small region. Moreover, by selecting electrons with a specific loss energy by a slit so as to image them, element distribution in specimen can be visualized (Elemental Mapping). From the intensity and the cross-section of inelastic scattering, the elemental ratio in the specimen is determined. From the fine structure of the absorption peak obtained in an EELS spectrum, the difference in bonding state and local electronic state can be detected. The distribution of elements in a specimen is clarified by selecting and imaging the electrons with a specific energy loss.

For our studies we used a Technai F-20 TEM operated at $200 \mathrm{KV}$ with a point resolution of $0.24 \mathrm{~nm}$ at the Center for Solid State Sciences, Arizona State University, Tampe,Arizona 85287-1704,USA.The EELS data was collected with $0.8 \mathrm{eV}$ energy resolution and 1 to $0.2 \mathrm{eV}$ dispersions.

\subsubsection{SQUID}

We used the Quantum Design MPMS 2 SQUID (Superconducting Quantum Interference Device) magnetometer for magnetic measurements of our samples. The SQUID is the most sensitive device available for measuring magnetic fields. The SQUID in the MPMS system does not detect the magnetic field from the sample directly. The measurement is performed in the MPMS instrument by moving the sample through the superconducting detection coils, which are located at the center of the superconducting magnet outside the sample chamber [123]. The detection coils are connected to the SQUID with superconducting wires, allowing the current from the detection coils to inductively couple to the SQUID sensor. As the sample moves through the detection coils, the magnetic moment of the sample 
induces an electric current in the detection coils. The detection coils, the connecting wires and the SQUID input coil form a closed superconducting loop. Any change in the magnetic flux in the detection coils produces a change in the persistent current in the detection circuit, proportional to the change in magnetic flux. Since the SQUID functions as highly linear current-to-voltage convertor, the variations in the current in the detection coils produce corresponding variations in the SQUID output voltage which are proportional to the magnetic moment of the sample. In a fully caliberated system, measurements of the voltage variation as the sample is moved through the detection coils provide a highly accurate measurement of the sample's magnetic moment. The system can be accurately caliberated using a small piece of material having a known mass and magnetic susceptibility.

The Quantum Design MPMS2 instrument with a superconducting magnet can be operated using fields upto 1 Tesla and measurements can be performed at temperatures ranging from 1.7 to $350 \mathrm{~K}$. The system makes use of a second order derivative coil configuration to eliminate effects of stray fields. The sample chamber is at low pressure of helium gas, which provides thermal contact with a flow of gas outside the sample chamber pipe that is used to change and stabilize the temperature. Over $4.5 \mathrm{~K}$ flow impedance and a gas heater take care of the temperature control. The temperature is homogenised around the sample space by copper wires.

For temperature dependence of magnetisation, $\mathrm{M}(\mathrm{T})$, two kinds of measurements were made. In the zero field cooled measurement (ZFC) the sample was cooled down to $5 \mathrm{~K}$ in the absence of a magnetic field and a constant magnetic field was applied during the warm up scan. In the field-cooled, (FC), measurements the sample was cooled from $310 \mathrm{~K}$ down to $5 \mathrm{~K}$ in the presence of the same constant magnetic field and then a warm up scan was performed in the same magnetic field. For the hysteretic loop, $M(H)$, measurements the samples were subjected to a magnetic field cycling between +1 Tesla and -1 Tesla and magnetisation was measured at a particular temperature.

\subsubsection{Ferromagnetic resonance spectroscopy (FMR)}

Ferromagnetic resonance provides experimental scientists with one of the most precise tools for investigation of ferromagnetic materials [124]. It is probably the most sensitive method for detecting ferromagnetic order as well as the possible existence of other magnetic species. FMR and electron paramagnetic resonance (EPR) are very sensitive and capable of detecting 
a total of $10^{13}$ spins. Valuable information regarding anisotropy and saturation magnetization can be also obtained.

Ferromagnetic resonance, or more precisely, resonance absorption of external electromagnetic radiation in ferromagnetics, belong to the category of phenomena dealt with RF spectroscopy. RF spectroscopy in wide sense, connects a large variety of interaction processes between the electromagnetic field and matter, both in the form of individual isolated atoms and molecules and in condensed phases viz., liquids and crystals. A characterstic feature of these processes, that they all occur with in the definite interval of wavelengths $(\sim 1 \mathrm{~mm}-10 \mathrm{~cm})$ or frequencies $\left(\sim 10^{9}-10^{11} \mathrm{~Hz}\right)$. The nearest predecessor of $\mathrm{RF}$ spectroscopy is the Zeeman effect. This effect is caused by the splitting of the energy levels of an isolated atom (Zeeman multiplets) by an external magnetic field $\mathrm{H}_{\mathrm{o}}$. The study of this splitting yields much information on the nature of the atoms and the electrons (spin, determination of $\mathrm{g}$ - factor, determination of selection rules, etc.). To every Zeeman multiplet there corresponds an admissible energy difference, one for each pair of levels ( $\mathrm{I}$ and k) of given multiplet. The frequency $v_{\mathrm{ik}}$ of the coresponding quanta which are absorbed or emitted in these transitions are determined by the well known expression for Bohr frequencies

$$
H v_{i k}=h \omega_{i k}=E_{i}-E_{k}=\Delta E_{i k}
$$

Where $\mathrm{h}=6.625 \times 10^{-27} \mathrm{erg} . \mathrm{sec}$ is plank's constant; $\omega_{\mathrm{ik}}$ are the angular Bohr frequencies; the energy difference $\Delta \mathrm{E}_{\mathrm{ik}}$ are calculated from the expression

$$
\Delta E_{i k}=g \mu_{B} \Delta m_{i k} H_{o}
$$

where $\mathrm{g}$ is the Lande factor linked to the ratio of the magnetic moment to the mechanical moment; $\Delta \mathrm{m}_{\mathrm{ik}}$ is the difference between the magnetic quantum numbers of states $\mathrm{i}$ and $\mathrm{k}$ of the given multiplet; $\mu_{\mathrm{B}}$ is Bohr magneton. If we restrict ourselves to the region of not too weak magnetic fields, than we can easily find from eq. (1) and (2) that the Bohr frequency $\left(\omega_{\mathrm{ik}}\right)$ for transition in question are $10^{9}-10^{11} \mathrm{~Hz}$, ie. high frequency range. Taking into consideration the selection rules and proceeding from eqn (1), (2), and the expression for $\mu_{\mathrm{B}}$ $(=\mathrm{eh} / 4 \pi \mathrm{mc} ; \mathrm{m}$ mass of electron, e charge of electron and $\mathrm{c}$ is velocity of light), we readily obtain a universal relationship between the resonance frequency and the external field $\mathrm{H}_{\mathrm{o}}$ :

$$
\omega_{\text {res }}=\gamma \mathrm{H}_{\mathrm{o}}
$$

where, $\gamma=4 \pi \gamma \mu_{\mathrm{B}} / \mathrm{h}=\mathrm{ge} / 2 \mathrm{mc}$

we observe from (3) and (4) that, to determine g-factor of the atomic system, it is sufficient, for instance, to fix the $\mathrm{H}_{\mathrm{o}}$ and to adjust the frequency to the resonance value $\omega_{\text {res }}$. Generally, 
however, the procedure is different: given a certain frequency $\omega_{0}$ of HF field, we find the resonance magnetic field $\mathrm{H}_{\text {res }}$ corresponding to the given frequency

$$
\mathrm{H}_{\mathrm{res}}=\omega_{\mathrm{o}} / \gamma
$$

Experimental procedure: In ferromagnetic resonance experiments, a mono - or polycrystalline, conducting or non-conducting ferromagnetic sample of some regular shape is placed in a resonance cavity or waveguide, where a weak alternating magnetic field of a fixed frequency $\left(v_{0}\right)$ is maintained $\left(v_{0} \sim 5 \times 10^{2}-10^{5}\right.$ megacycles). Perpendicular to this HF field $h(t)$, a strong, homogeneous magnetizing field $\mathrm{H}_{\mathrm{o}}$ is applied, generally bringing the sample close to saturation. If the condition $h \nu_{\mathrm{o}}=\mathrm{g} \mu_{\mathrm{B}} \mathrm{H}_{\mathrm{eff}}$ is satisfied, than the oscillating field will induce transition between neighbouring Zeeman sublevels of the ferromagnetic system separated from each other by $g \mu_{\mathrm{B}} \mathrm{H}_{\text {eff }}\left(\mathrm{H}_{\text {eff }}\right.$ is the effective internal field in the ferromagnetic material, $\mu_{\mathrm{B}}$ is the Bohr magneton, and $\mathrm{g}$ the spectroscopic splitting factor, analogous to the Lande' g-factor in optical spectroscopy). In experimental studies of ferromagnetic resonance, the frequency of the high frequency radiation is kept constant for purely technical reasons, while the intensity of the external field is varied over certain limits about $\mathrm{H}_{0}$, thus changing the effective field $\mathrm{H}_{\mathrm{eff}}$. At certain point resonance absorption of $\mathrm{HF}$ field energy by the sample occurs leading to a change in $\mathrm{Q}$ (the $\mathrm{Q}$ of a resonance cavity is the ration of the maximum power of the oscillations in the cavity to the average power loss per cycle) of the cavity, which can be determined experimentally. 


\section{Results and discussion}

Mn doped ZnO: Despite theoretical prediction of a possibility of room temperature ferromagnetism in p-type $\mathrm{Mn}$ doped $\mathrm{ZnO}$ [9], no report of the confirmation of the same was reported until long. Attempts to make $\mathrm{Mn}$ doped $\mathrm{ZnO}$ based DMS resulted in paramagnetic or spin glass behaviour or a low temperature ferromagnetic ordering.

We reported the first observations of ferromagnetism above room temperature for dilute ( $<4$ at.\%) Mn-doped $\mathrm{ZnO}$ [53]. The $\mathrm{Mn}$ is found to carry an average magnetic moment of $0.16 \mu \mathrm{B}$ per ion. Our ab initio calculations find a valance state of $\mathrm{Mn} 2+$ and that the magnetic moments are ordered ferromagnetically, consistent with the experimental findings. We have obtained room-temperature ferromagnetic ordering in powder form,bulk pellets, and in transparent films $2-3 \mu \mathrm{m}$ thick, of the same material. The unique feature of our sample preparation was the low-temperature processing. This capability to fabricate ferromagnetic $\mathrm{Mn}$-doped $\mathrm{ZnO}$ semiconductors promises new spintronic devices as well as magneto-optic components.

Following our work several other groups have reported similar phenomena in $\mathrm{Mn}$ doped $\mathrm{ZnO}$ system.

\subsection{An overview of the papers included in the thesis}

Initially, we too followed the conventional high temperature processing of $\mathrm{Mn}$ doped $\mathrm{ZnO}$ samples and found absence of ferromagnetic ordering at room temperature. However, a ferromagnetic phase was formed with transition temperature below $45 \mathrm{~K}$ as shown in the Fig.13. The Magnetisation curve at $5 \mathrm{~K}$ showed a superposition of two phases (Inset Fig.13). 


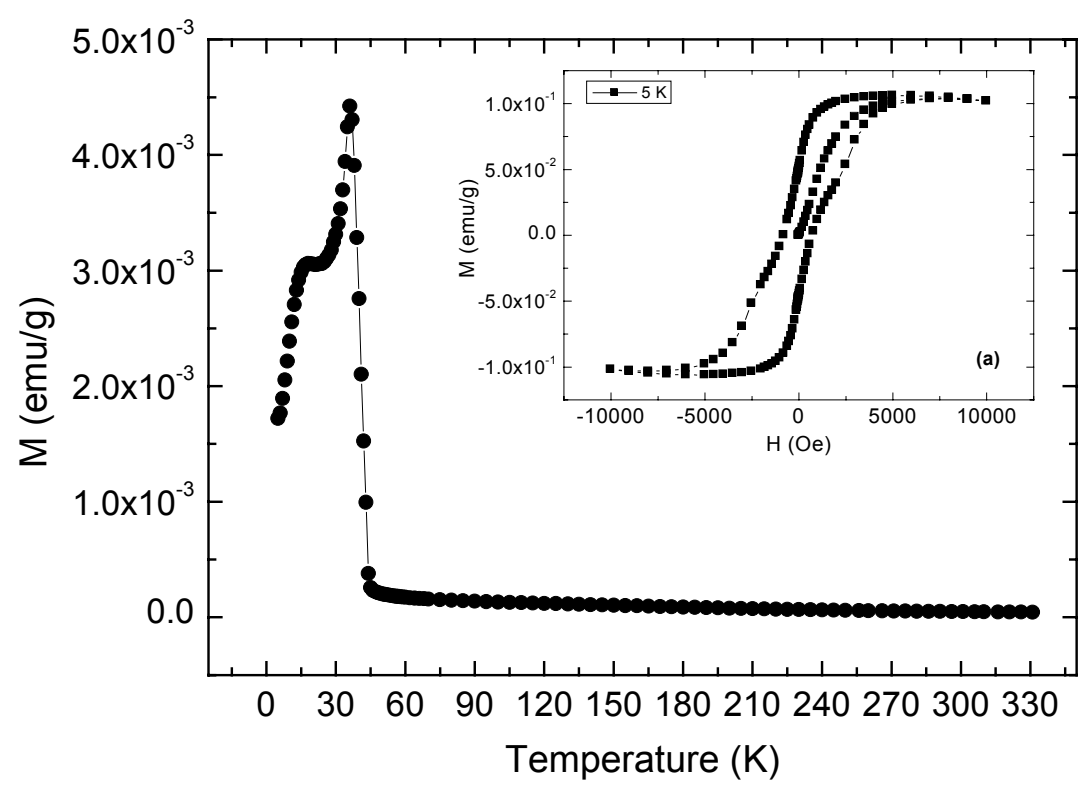

Fig-13 $M(T)$ at 50 Oe for Zn1-x MnxO (x 0.02) ceramic pellet sintered at temperature of $9000 \mathrm{C}$. Inset (a) shows the $M(H)$ at $5 \mathrm{~K}$

Thereafter, we tried sintering our samples at various temperatures ranging from $500^{\circ} \mathrm{C}$ to $900^{\circ} \mathrm{C}$, and found ferromagnetic ordering with a Tc $>425 \mathrm{~K}$ in samples sintered below $700^{\circ} \mathrm{C}$. Sintering above $700^{\circ} \mathrm{C}$ resulted in the suppression of ferromagnetic ordering and enhanced paramagnetic contribution. Similar effect was also observed on increasing the concentration of $\mathrm{Mn}$. We tried low temperature sintering following the fact that $\mathrm{ZnO}$ doped with a TM will go magnetic only if it is p-type. High temperature processing might lead to increased n-type carriers in the system.

Once we obtained ferromagnetic ordering at room temperature in $\mathrm{Mn}$ doped $\mathrm{ZnO}$, as measured from SQUID magnetometer, we made EDS analyses, and elemental mapping on our samples to verify the actual $\mathrm{Mn}$ concentration incorporated into the $\mathrm{ZnO}$ lattice without distorting its wurtzite structure. We found that though Mn was distributed uniformly in our low temperature sintered samples but its concentration was much less $(\sim 0.3$ at $\%)$ than the nominal concentration ( 2 at \%) used for doping $\mathrm{ZnO}$. Using this value for $\mathrm{Mn}$ concentration we estimated the average magnetic moment of $\sim 0.16 \mu \mathrm{B}$ per ion. This number was found to vary from one sample batch to another depending upon the precursor source and the environment. This is so because the carrier concentration (either p-or n-type) in the sample seems to be sensitive to these factors, which might affect the magnetization, if the carrier induced ferromagnetic ordering exist in $\mathrm{Mn}$ doped $\mathrm{ZnO}$. For high temperature sintered 
samples $\left(<700^{\circ} \mathrm{C}\right)$, Mn was found to form clusters, which pointed towards the loss of room temperature ferromagnetic ordering in these samples as $\mathrm{Mn}-\mathrm{Mn}$ direct interactions are antiferromagnetic. The X-ray diffraction pattern for $\mathrm{Mn}$ doped $\mathrm{ZnO}$ bulk sintered below $700^{\circ} \mathrm{C}$ matched with that of pure $\mathrm{ZnO}$ without any indication of a secondary phase. For $900^{\circ} \mathrm{C}$ sintered pellet, other oxides of Mn along with a MnZn compound showed up in the XRD spectra. Such secondary phases were also observed in the bulk samples with a higher concentration of manganese.

After initial characterisation of the bulks we made $\mathrm{MnZnO}$ films using PLA. The nominal composition of the target used for ablation was kept $10 \%$ following the results from composition analyses that actual Mn concentration in the bulk to be ablated would be less. The films were grown on fused quartz (pure $\mathrm{SiO}_{2}$ ) substrate at a substrate temperature of $400^{\circ} \mathrm{C}$ and $600^{\circ} \mathrm{C}$. Elemental analyses of the films showed homogeneous distribution of $\mathrm{Mn}$, and its concentration was found to be $2.21 \%$ and $4.56 \%$ respectively. The XRD confirmed their c-axis orientation. And the magnetic characterization showed hysteresis at room temperature for both the film samples.

Our preliminary results were confirmed further by FMR spectroscopy. The obtained FMR signal showed the ferromagnetic transition temperature to be well above $425 \mathrm{~K}$ for the low temperature processed bulks and thin films [53, 125].

Selected area electron diffraction (SAED) patterns and High resolution images taken on our thin film samples revealed an ordered hexagonal structure of $\mathrm{ZnO}$ and absence of any clusters in the sample. The presence of $\mathrm{Mn}$ in the lattice, in $\mathrm{Mn}^{2+}$ valence state, was confirmed by the EELS measurements from the same area.

\section{Further investigations}

All these results proved to be guidelines for studying the mechanism of observed magnetic properties in our samples. In order to find an explaination as to what happens on heating the same calcined samples to temperatures higher than $700^{\circ} \mathrm{C}$, we processed our precursors $\mathrm{ZnO}$ and $\mathrm{MnO}_{2}$ used in the present study in a similar way as we did our $\mathrm{Mn}$ doped $\mathrm{ZnO}$ samples.

It was found that $\mathrm{MnO}_{2}$ is sensitive to processing temperatures and decomposes to its lower oxides when heated above $500^{\circ} \mathrm{C}$. Our results showed that the $\mathrm{MnO}_{2}$ pellet sintered at $500^{\circ} \mathrm{C}$ was still antiferromagnetic. But the $\mathrm{MnO}_{2}$ pellet sintered at $900^{\circ} \mathrm{C}$ showed a low temperature ferro/ferri-magnetic ordering. The temperature dependence of magnetisation at 50 Oe for the $\mathrm{MnO}_{2}$ pellet sintered at $900{ }^{\circ} \mathrm{C}$ showed a sharp fall in magnetization at $42 \mathrm{~K}$. Hysteresis, 
which was present at $5 \mathrm{~K}$ in the $\mathrm{M}(\mathrm{H})$ curve disappeared above $42 \mathrm{~K}$. Thus a ferriferromagnetic phase with $\mathrm{Tc}<45 \mathrm{~K}$ was formed. The temperature dependence of magnetisation for $\mathrm{MnO}_{2}$ sintered at $900^{\circ} \mathrm{C}$ is shown below.

The trend was similar to the temperature dependence of magnetization obtained for our $\mathrm{Mn}$ doped $\mathrm{ZnO}$ pellets sintered at high temperatures. From various studies done on $\mathrm{MnO}_{2}$ we understand that heating of $\mathrm{MnO}_{2}$ between 500 and $1100^{\circ} \mathrm{C}$ in air results in its decomposition to number of lower oxides out of which $\mathrm{Mn}_{3} \mathrm{O}_{4}$ is ferro/ferri-magnetic with a Tc of $<45 \mathrm{~K}$. Hence the possible source of low temperature magnetic phase could be $\mathrm{Mn}_{3} \mathrm{O}_{4}$. Another candidate responsible for this ferromagnetic ordering could be phases of ( $\mathrm{Zn} \mathrm{Mn}) \mathrm{Mn}_{2} \mathrm{O}_{3}$ which are known to exhibit low temperature ferromagnetic ordering [126].

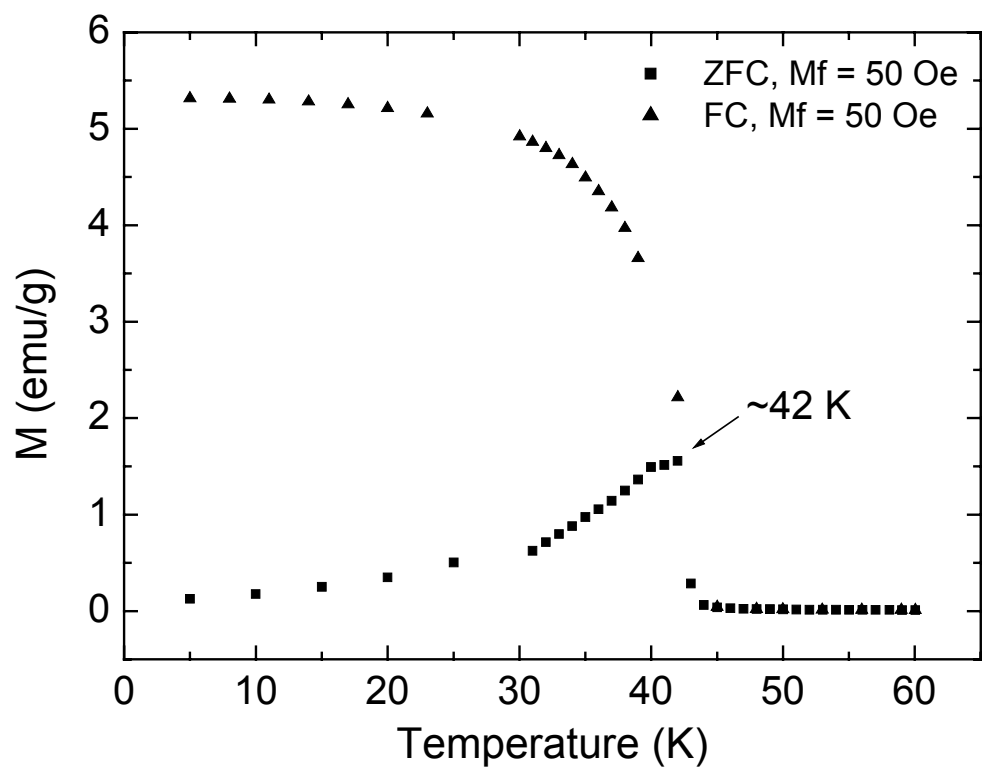

Fig-14 M (T) at 50 Oe in the zero field cooled (ZFC) and field cooled (FC) state for $\mathrm{MnO}_{2}$ pellet sintered at $900^{\circ} \mathrm{C}$

The disappearance of room temperature ferromagnetic ordering can also be explained by the fact that $\mathrm{ZnO}$ tends to lose oxygen when heated to high temperatures and thus becomes $\mathrm{n}$ type. The lattice shrinkage seen in the XRD pattern for the pure $\mathrm{ZnO}$ powder sintered at various temperatures confirmed the loss of oxygen. It can be pointed at this point that the colour of our pellets processed at low temperature is whitish and more like stoichiometric $\mathrm{ZnO}$, but changes to yellow on processing at higher temperatures further suggesting an increase in the n-type carriers. 
Hence the low temperature ferromagnetic ordering obtained in high temperature sintered samples is attributed to the ferrimagnetic oxide of $\mathrm{Mn}$ and the secondary ferromagnetic phase of $\mathrm{Mn}$ and $\mathrm{Zn}$ while the disappearance of the same to formation of Mn clusters and increased n-type conductivity of $\mathrm{ZnO}$.

Thus we established that low temperature processing is indeed the key to obtain room temperature ferromagnetic ordering in $\mathrm{Mn}$ doped $\mathrm{ZnO}$ samples, both because it prevents, formation of secondary phases and the samples from becoming n-type.

We further investigated the problem of low magnetic moment of $0.16 \mathrm{uB} / \mathrm{Mn}$ obtained for our samples as compared to $5 \mu_{\mathrm{B}}$ per Mn for the fully polarized state. Clearly in our samples all the Mn spins don't contribute to magnetization and are blocked. This can be well understood from the fact that the spin dependent hybridization between host $\mathrm{p}$ and $\mathrm{Mn} \mathrm{d}$ orbitals leads to antiferromagnetic interactions between the Mn moments, which can only be overcompensated by hole mediated long range ferromagnetic interactions. Hence lack of sufficient carriers (holes) to mediate long range ferromagnetic ordering leads to decrease in effective Mn concentration, hence the magnetic moment.

To verify the above, we prepared $\mathrm{Zn}_{1-\mathrm{x}} \mathrm{Mn}_{\mathrm{x}} \mathrm{O}$ samples with different concentration of $\mathrm{Mn}$ and studied the Mn concentration dependence of ferromagnetic and paramagnetic phase. We found that whereas the paramagnetic term increases almost linearly with the increase in $\mathrm{Mn}$ concentration, the ferromagnetic phase remained more or less the same for all $\mathrm{Mn}$ concentrations.

This shows lack of sufficient carriers to mediate ferromagnetic exchange between localized Mn spins. Since no additional carriers are provided, their concentration is supposed to be fixed in the samples because processing conditions and precursors were kept same for all the samples. Addition of more and more Mn atoms merely adds antiferromagnetically interacting spins, thus increasing the paramagnetic contribution at room temperature.

This is hence crucial to have sufficient carrier density in the samples. We therefore, doped our $\mathrm{Zn}_{1-\mathrm{x}} \mathrm{Mn}_{\mathrm{x}} \mathrm{O}$ samples with different concentration of $\mathrm{Cu}$, which has been shown by researchers as a possible codopant [114]. Comparing the magnetization of $\mathrm{Zn}_{1-\mathrm{x}} \mathrm{Mn}_{\mathrm{x}} \mathrm{O}$ and $\mathrm{Zn}_{1-\mathrm{x}-}$ ${ }_{y} \mathrm{Mn}_{\mathrm{x}} \mathrm{Cu}_{\mathrm{y}} \mathrm{O}$ samples from the same initial batch, we found a considerable enhancement in magnetization after codoping with $\mathrm{Cu}$. The XRD spectra of these $\mathrm{Zn}_{1-\mathrm{x}-\mathrm{y}} \mathrm{Mn}_{\mathrm{x}} \mathrm{Cu} \mathrm{y}$ powders calcined at $400^{\circ} \mathrm{C}$ for $8 \mathrm{hrs}$ matched with that of host $\mathrm{ZnO}$. The slight shift in peaks was observed which is indicative of the fact that $\mathrm{Cu}$ is incorporated into the lattice. No peak for manganese oxides or any other secondary phase was detected. However, we found peaks for $\mathrm{CuO}$ in the sample. Thus the $\mathrm{CuO}$ used for doping $\mathrm{Zn}_{1-\mathrm{x}} \mathrm{Mn}_{\mathrm{x}} \mathrm{O}$ was not dissolved completely. 
This is not critical since $\mathrm{CuO}$ is antiferromagnetic below $230 \mathrm{~K}$ thus can not be a source of increased ferromagnetic ordering in the $\mathrm{Cu}$ doped samples. It is however important to be able to achieve higher solubility of $\mathrm{Cu}$ into $\mathrm{Zn}_{1-\mathrm{x}} \mathrm{Mn}_{\mathrm{x}} \mathrm{O}$ as it might enhance magnetization further. The EDS analyses done on the $\mathrm{Zn}_{1-\mathrm{x}-\mathrm{y}} \mathrm{Mn}_{\mathrm{x}} \mathrm{Cu}_{\mathrm{y}} \mathrm{O}$ calcined powder also showed absence of $\mathrm{MnO}_{2}$ precursor as it is but $\mathrm{CuO}$ could be seen undissolved in some areas. Therefore, the actual concentration of $\mathrm{Cu}$ incorporated into the host lattice was much less than that used for doping. We also deposited thin films of $\mathrm{Zn}_{1-\mathrm{x}-\mathrm{y}} \mathrm{Mn}_{\mathrm{x}} \mathrm{Cu}_{\mathrm{y}} \mathrm{O}$ by $\mathrm{rf}$ magnetron sputtering technique. Again considering the fact that the actual concentration of dopant $(\mathrm{Mn}$ and $\mathrm{Cu})$ in the sample is less, we made a sputtering target of $\mathrm{Zn}(\mathrm{Mn}, \mathrm{Cu}) \mathrm{O}$ with nominal concentration of $\mathrm{Mn}$ and $\mathrm{Cu}$ to be $10 \%$. A dense sputtering target was made by spark plasma sintering (SPS) technique. As obtained target was sputtered at a rf power of $100 \mathrm{~W}$, to obtain the $\mathrm{Zn}(\mathrm{MnCu}) \mathrm{O}$ thin films. The XRD measurement showed a single phase film with preferred c-axis orientation, and clearly indicate the high solubility of $\mathrm{Cu}$ and $\mathrm{Mn}$ in thin films as compared to bulk samples. The magnetic measurement by SQUID clearly showed the existence of above room temperature ferromagnetic ordering in $\mathrm{Zn}(\mathrm{MnCu}) \mathrm{O}$ thin films.

We also studied the electronic structure of $\mathrm{Mn}$ doped $\mathrm{ZnO}$ thin film using X-ray absorption spectroscopy (XAS), X-ray emission spectroscopy (XES) and resonant inelastic scattering. The $\mathrm{O} 2 \mathrm{p}$ absorption and $\mathrm{K}$-emission spectra revealed a strong hybridization between Mn 3d and O 2p states. The Mn doping enhances upper valence band, thus reducing the band gap size. The same results were also obtained theoretically using LDA calculations.

\section{Other promising magnetic semiconductors}

While the above investigations were being carried out, we explored other candidate materials for DMS and found ferromagnetic ordering in

a) $\mathrm{ZnO}$ doped with $\mathrm{Cu}$

b) $\mathrm{GaP}$ doped with $\mathrm{Cu}$

c) $\mathrm{ZnS}$ doped with $\mathrm{Mn}$

d) $\mathrm{CdS}$ doped with $\mathrm{Mn}$

In the present thesis only $\mathrm{ZnO}$ doped with $\mathrm{Cu}$ and $\mathrm{GaP}$ doped with $\mathrm{Cu}$ are included.

\section{ZnO doped with Copper}

We doped $\mathrm{ZnO}$ with $\mathrm{Cu}$ instead of manganese and studied it magnetic properties and found that $\mathrm{Zn}_{1-\mathrm{x}} \mathrm{Cu}_{\mathrm{x}} \mathrm{O}$ is ferromagnetic above room temperature. Initial characterization using XRD and electron diffraction and EDS showed incorporation of $\mathrm{Cu}$ in the lattice, but undissolved 
$\mathrm{CuO}$ was also present. As stated earlier, presence of unreacted $\mathrm{CuO}$ does not affect the ferromagnetic ordering as it is antiferromagnetic. The presence of ferromagnetic ordering was detected by magnetic measurements performed using SQUID magnetometer and ferromagnetic resonance spectroscopy.

We also investigated the electronic structure of $\mathrm{Cu}$ doped $\mathrm{ZnO}$ by XAS and XES.

\section{GaP doped with copper}

We discovered another unusual room temperature ferromagnetic semiconductor that is Gallium phosphide doped with copper. The ferromagnetic ordering in bulk GaP doped with copper is detected by SQUID magnetometry and ferromagnetic resonance. The Tc was estimated to be above $700 \mathrm{~K}$. The important features of this observation is relatively simple sintering process for making the material and significantly higher Curie temperature.

Further extensive investigation like neutron scattering experiments are in progress.

\subsection{Conclusions}

Ferromagnetism in $\mathrm{Mn}$ doped $\mathrm{ZnO}$, powders, bulks as well as thin films could be obtained at and above room temperature by tailoring the process parameters. The key to observed phenomenon in this system is the low temperature processing, which prevents formation of clusters, secondary phases and the host $\mathrm{ZnO}$ from becoming n-type. The ferromagnetism is indeed carrier mediated and can be enhanced by additional doping with holes, thus co-doping by an acceptor impurity like $\mathrm{Cu}$. Ferromagnetic transition temperature was found to be well above $425 \mathrm{~K}$ by Ferromagnetic Resonance spectroscopy. X-ray diffraction confirmed the absence of secondary phases in the low temperature sintered samples. High Resolution Transmission Electron microscopy along with Selected Area Electron Diffraction and Electron Energy Loss Spectroscopy showed uniform distribution of magnetic dopant atoms into the hexagonal structure of the host $\mathrm{ZnO}$ without distorting it. The electronic structure studies done by Soft X-ray spectroscopies indicated p-d exchange interactions, which is characteristic of DMS. It also showed that Mn doping enhanced the upper valence band, thus reducing the band gap size. $\mathrm{Cu}$ doping increased the magnetisation in $\mathrm{Mn}$ doped samples. Surprisingly $\mathrm{Cu}$ doping alone in $\mathrm{ZnO}$ resulted in room temperature ferromagnetic ordering. 


\section{Suggestions for Future studies \\ The journey continues!}

The studies presented in the thesis were mainly focussed on powders and bulk material, though the phemomena has been demonstrated for thin films also. In future we would concentrate on fabrication and characterisation of thin films. Apart from structural characterization, there are three routes to obtain information whether the doping material is an intrinsic part of the material. One is through Hall measurements, by identifying Ordinary Hall effect and Anomalous Hall effect, another is through ferromagnetic resonance (FMR) and the third is through magnetic circular dichorism (MCD), which is directly due to the sp-d interaction identifying it as a DMS. Besides that we would focus on developing new materials for making dilute magnetic semiconductors.

Towards applications, our efforts will be for the fabrication of Spin-FET with ZnO based DMS. As the carrier concentration in $\mathrm{ZnO}$ can be controlled optically [13, 127, 128, 129], so this system can allows us to fabricate "Photon controlled devices such as photo-magnets and sensors", in which the magnetic property of $\mathrm{ZnO}$ will be controlled by the application of UV light.

Search for new materials with higher saturation magnetization, and controllable via carrier induced ordering process will always be a part of the on going studies. 
1. S. A. Chambers, Materials today (April 2002) 34

\section{References}

2. H. Ohno, F.Matsukura and Y,Ohno, JSAP International, 5 (2002), 4

3. S.J. Pearton, C.R. Abernathy, D.P. Norton, A.F. Hebard, Y.D. Park, L.A. Boatner, J.D. Budai, Mat Sc. And Engg., R 40 (2003), 137

4. S. Das Sarma, American Scientist, 89 (2001) 516

5. H. Ohno, Science, 281 (1998), 951

6. J.K. Furdyna, J. Appl. Phys., 64 (1988) R29

7. S. Koshihara, A. Oiwa, M. Hirasawa, S. Katsumoto, Y. Iye, C. Urano, H. Takagi , H. Munekata, Phys. Rev. Lett.,78 (1997) 4617

8. M. Nazmul, S.Ahsan, \& Tanaka, Cond-mat (2003), 0208299

9. T. Dietl, H.Ohno, F. Matsukura, J.Cibert. \& D. Ferrand, Science, 287 (2000), 1019

10. P. A. Stampe, R. J. Kennedy, X.Yan, J. S. Parker, J. Appl. Phys., 92, (2002) 7114

11. S. A. Chambers, Appl.Phys. Lett., 82, (2003) 1257

12. J.H. Kim, H. Kim, Y.E. Ihm, \& W.K. Choo, J. Appl. Phys., 92, (2002). 6066

13. P. Sharma, K. Sreenivas, and K. V. Rao, J. Appl. Phys., 93, (2003) 3963

14. S.N. Khanna, B.K. Rao, P. Jena, M. Knickelbein, Chemical Phys. Letts., 378 (2003) 374

15. H. Sato, Y. Kawamuraa, T. Ogawaa, Y. Murakamib, H. Ohsumic, M. Mizumakic, and N. Ikeda, Physica B, 329-333 (2003) 757.

16. S. Das. Sarma, J. Fabian, X.Hu, I. Zutic, Solid State Comm, 119(2001) 207

17. K. Tanahashi, A. Kikukawa, Y. Takahashi, Y. Hosoe, M. Futamoto, J. of Mag. and Mag. Materials, 242-245 (2002) 325.

18. M. L. Roukes, Nature, 411 (2001) 747

19. M. N. Baibich, J. M. Broto, A. Fert, F. Nguyen Van Dau, F. Petroff, P. Etienne, G. Creuzet, A. Friederich, and J. Chazelas, Phys. Rev. Lett. 61, (1988) 2472.

20. S. S. Parkin, "Gaint magnetoresistance in magnetic nanostructures", in Annual Review of Materials Science, B. W. Wessels, Ed. Palo Alto, CA: Annual Reviews Inc., 25 (1995) 357.

21. S. Parkin, X. Jiang, C. Kaiser, A. Panchula, K. Roche, and M. Samant, Proceedings of the IEEE, 91(5) (2003) 661.

22. S. Das Sarma, J. Fabian, X. Zu, I. Zutic, Superlattices and Microstructures, 27 (5/6), (2000) 289.

23. Datta and Das, Appl. Phys. Lett., 56, (1990) 665

24. Y. D. Park, B.T. Jonker, B. R. Bennet, G, Itzkos, M. Furis, G. Kioseoglou and A. Petrou, Appl. Phys. Lett., 77 (2000) 3989

25. Ohno et al. Nature 408, (2000) 944.

26. G. A. Medvedkin, T. Ishibashi, T. Nishi, K Hayata, Jpn. J. Appl. Phys., 39 (2000) L949

27. H. Ohno, F. Matsukura, Solid state. Comm., 117(2001) 179

28. A. Haury, A. Wasiela, A. Arnoult, J. Cibert, S. Tatarenko, T. Dietl, Y. Merle d'Aubigne, Phys. Rev. Lett., 79 (1997) 511

29. T. Story, R.R. Galazka, R.B. Frankel, P.A. Wolff, Phys. Rev. Lett., 56 (1986) 777

30. Y. Fukuma, H. Asada, J. Miyashita, N. Nishimura, and T. Koyanagi, Journ. Of Appl. Phys., 93 (2003) 7667

31. H. Munekata, H. Ohno, S. Von Molnar, A. Segmuller, L.L. Chang, L. Esaki, Phys. Rev. Lett., 63 (1989) 1849, 
32. H. Ohno, H. Munekata, T. Penny, S. Von Molnar, L.L. Chang, Phys. Rev. Lett., 68 (1992) 2664

33. H. Ohno, A. Shen, F. Matsukura, A. Oiwa, A. Endo, S. Katsumoto, and Y. Iye. Appl. Phys. Lett, 69 (1996) 363

34. Y. D. Park, A.T. Hanbicki, S.C. Erwin, C.S. Hellberg, J.M. Sullivan, J.E. Mattson, T.F. Ambrose, A. Wilson, G. Spanos, B.T. Jonker, Science, 95 (2002) 651

35. Y. Matsumoto, M. Murakami, T. Shono, T. Hasegawa, T. Fukumura, M. Kawasaki, P. Ahmet, T. Chikyow, Shin-ya Koshihara, H. Koinuma, Science, 291 (2001) 854

36. K. Ueda , Hitoshi Tabata, and Tomoji Kawai, Appl. Phys. Lett., 79 (2001), 988

37. H. Saeki, H. Tabata, T. Kawai, Solid State Commun., 120 (2001), 439

38. S.J. Pearton, M.E. Overberg, C.R. Abernathy, N.A. Theorodopoulou, A.F: Hebard, S.N.G. Chu, A. Osinski, V.Fuflyigin, L.D. Zhu, A.Y. Polyakov, R.G. Wilson, J. Appl. Phys., 92 (2002), 2047

39. G. A. Medvedkin, K. Hirose, T. Ishibashi, T.Nishi, V.G. Voevodin,K.Sato, J. Cryst. Growth 236 (2002), 609

40. H. Hori, S.Sonoda,T. Sasaki, Y. Yamamoto, S. Shimizu, K.-I. Suga, K. Kindo, Physica B, 324 (2002), 142

41. S. Choi, G.B. Cha, S.C. Hong, S. Cho, Y. Kim, J.B.Ketterson, S.-Y. Jeong, G.-C. Yi, Solid State Commun., 122 (2002), 165

42. N. Theodopoulou, A.F. Hebard, M.E. Overberg, C.R. Abernathy, and S.J. Pearton, S.N.G. Chu, R.G. Wilson, Phys. Rev. Lett., 89 (2002), 107203

43. M. Hashimoto, Y-K. Zhou, M. Kanamura, H. Asahi, Solid State Commun., 122 (2002), 37

44. X. Chen, M. Na, M. Cheon, S. Wang, H. Luo, B. D. McCombe, Appl. Phys. Lett., 81 (2002), 511

45. S.-J. Han, J. W. Song, C.-H. Yang, S. H. Park, J.-H. Park, and Y. H. Jeong, Appl. Phys. Lett., 81 (2002), 4212

46. N. Teraguchi, A. Suzuki, Y. Nanishi, Y-K. Zhou, M. Hashimoto, H. Asahi, Solid State Commun., 122 (2002), 651

47. H.C. Jeon Y.S. Jeong, T.W. Kang, T.W. Kim, K.J. Chung, K.J. Chung, W. Jhe, S.A. Song, Adv. Mater., 14 (2002), 1725

48. S.G. Yang, A. B. Pakhomov, S. T. Hung, and C. Y. Wong, Appl. Phys. Lett., 81 (2002), 2418

49. H. Saito H. Saito V. Zayets, S. Yamagata, and K. Ando Phys. Rev. Lett., 90 (2003), 207202

50. J. S. Lee, J.D. Lim, Z.G. Khim, Y.D. Park, S.J. Pearton, S.N.G. Chu, J. Appl. Phys., 93 (2003), 4512

51. W. Wang et al., J. Supercond., 16 (2003), 155

52. S. B. Ogale, R. J. Choudhary, J. P. Buban, S. E. Lofland,S. R. Shinde, S. N. Kale, V. N. Kulkarni, J. Higgins, C. Lanci,J. R. Simpson, N. D. Browning, S. Das Sarma, H. D. Drew, R. L. Greene, and T. Venkatesan, Phys. Rev. Lett., 91 (2003), 077205

53. P. Sharma A. Gupta, K.V. Rao, F.J. Owens, R.Sharma, R.Ahuja, J. M. Osorio Guillen, B. Johansson. G.A. Gehring, Nature Materials, 2, (2003), 673

54. P.V. Radovanovic and D.R. Gamelin, Phys. Rev. Lett., 91, (2003), 157202

55. S. Das Sarma, E.H. Hwang, A. Kaminski,Solid State Commun,127 (2003), 99

56. T. Dietl, Cond-mat, 18 (2003), 0306479

57. T. Dietl, Cond-mat, 16 (2002), 0201282

58. J. De Boek, R. Oesterholt, a. Van Esch, H. Bender, C. Bruynseraede, C. Van Hoof, Appl. Phys. Lett, 68 (1996), 2744

59. H. Ohno, J. of Magn. and Mag. Mat., 200 (1999)110 
60. T. Hayashi, M. Tanaka, T. Nishinaga, H. Shimada, H. Tsuchiya and Y. Ohtsuka, J. Cryst. Growth, 175/176 (1997), 1063.

61. Y. Nishikawa, Y. Satoh and J. Yoshino, 1997, Second Symposium on Physics and Application of Spin Related Phenomena in Semiconductors, (27-28 Jan 1997, Sendai, Japan), 122.

62. J. Sadowski, J. Domagala, J. Bak-Misiuk, K. Sqiatek, J. Kanski, L.Ilver and H. Oscarsson, Acta Physica Polonica A, 94 (1998), 509.

63. R.K.Kawakami, E. Hohnston-Halperin, L. F. Chen, M. Hanson, N.Guébels, J. S. Speck, A. C. Gossard, and D. D. Awshalom, Appl. Phys. Lett., 77, (2000), 2379.

64. X. Liu, Y. Sasaki and J. K. Furdyna, Appl. Phys. Lett., 79 (2001), 2414.

65. H. Nojiri, M. Motokawa, S. Takeyama, F. Matsukura, H. Ohno, Physica B, 256-258 (1998), 569

66. H. Shimizu, T. Hayashi, T. Nishinaga and M. Tanaka, Appl. Phys.Lett. 74, (1999), 398.

67. G.M. Schott, W. Faschinger and L. W. Molenkamp, Appl. Phys.Lett., 79, (2001), 1807.

68. Matsukura, F., H. Ohno, A. Shen and Y. Sugawara, Phys. Rev. B, 57 (1998b), R2037.

69. K. Takamura, F. Matsukura, Y. Ohno and H. Ohno, J. Appl. Phys., 89 (2001), 7024

70. A. Shen, H. Ohno, F. Matsukura, Y. Sugawara, N. Akiba, T. Kuroiwa, A. Oiwa, A. Endo, S. Katsumoto and Y. Iye, J. Cryst. Growth, 175/176 (1997a), 1069.

71. S. J. Potashnik, K. C. Ku, S. H. Chun, J. J. Berry, N. Samarth and P.Schiffer, Appl. Phys. Lett., 79 (2001), 1495.

72. Y-J.Zhao, W. T. Geng, K. T. Park and A. J. Freeman, Phys.Rev. B 64 (2001a), 035207.

73. Slupinski, T., H. Munekata and A. Oiwa, Appl. Phys. Lett., 80 (2002a), 1592.

74. N. Theodopoulou, A.F. Hebard, M.E. Overberg, C.R. Abernathy, and S.J. Pearton, S.N.G. Chu, R.G. Wilson, Phys. Rev. Lett., 89 (2002), 107203

75. N. Theodopoulou, A.F. Hebard, S.N.G. Chu, M.E. Overberg, C.R. Abernathy, S.J. Pearton, R.G. Wilson, J.M. Zavada, Journ. of Appl. Phys., 91 (2002), 7499

76. M.E. Overberg, B.P. Gila, C.R. Abernathy, S. J. Pearton, N. A. Theodopoulou, K.T. McCarthy, S.B. Arnason, Appl. Phys. Lett., 79 (2001), 3128

77. M. Zajac, R. Doradzinski, J. Gosk, J. Szczytko, M. Lefeld-Sosnowska, M. Kaminska, and A. Twardowski, M. Palczewska, E. Grzanka, W. Gebicki, Appl. Phys. Lett., 78 (2001), 1276

78. W. Gebicki and J. Strzeszewski, G. Kamler, T. Szyszko, and S. Podsiadlo, Appl. Phys. Lett., 76, (2000), 3870

79. M. Zając, M., J. Gosk, M. Kamińska, A. Twardowski, T. Szyszko and S. Podsiadło, Appl. Phys. Lett., 79 (2001b), 2432.

80. S. Kuwabara, K. Ishii, S. Haneda, T. Kondo, and H. Munekata, Physica E, 10 (2001a), 233.

81. S. Kuwabara, T. Kondo, T. Chikyow, P. Ahmet, and H. Munekata, Jpn. J. Appl. Phys., 40 (2001b), L727.

82. M.E. Overberg, C.R. Abernathy, S. J. Pearton, N. A. Theodopoulou, K.T. McCarthy, F. Hebard, Appl. Phys. Lett., 79 (2001), 1312

83. S. Sonoda, S. Shimizu, T. Sasaki, Y. Yamamoto and H. Hori, cond-mat., (2001), 0108159

84. H. Akinaga, S. Németh, J. De Boeck, H. Bender, G. Borghs, H. Ofuchi,and M. Oshima, Appl. Phys. Lett., 77, (2000b), 4377.

85. Y-J. Zhao, W. T. Geng, A. J. Freeman and T. Oguchi, Phys. Rev. B, 63 (2001b), 201202. 
86. P. Mahadevan, P and A. Zunger, Phys. Rev. Lett., 88 (2001), 047205.

87. H.Akai, Phys. Rev. Lett., 81 (1998), 3002.

88. T. Fukumura, Y.Yamada, H.Toyosaki, T.Hasegawa, H.Koinuma, M. Kawasaki, Condmat, (2003), 0305435

89. Y. Matsumoto, M. Murakami, T. Shono, T. Hasegawa, T. Fukumura, M. Kawasaki, P. Ahmet, T. Chikyow, Shin-ya Koshihara, H. Koinuma, Science, 291 (2001), 854

90. G. C. Han, Y.H. Wu, M.Tay, K.B. Li, Z.B. Guo, T.C. Chong, Journ. of Magn And Mag. Mat., 268 (2004), 159.

91. S. A. Chambers and S. Thevuthasan, R. F. C. Farrow, R. F. Marks, J. U. Thiele, L. Folks, M. G. Samant, and A. J. Kellock, N. Ruzycki, D. L. Ederer, and U. Diebold, Appl. Phys. Lett., 79(2001), 3467.

92. S. A. Chambers, T. Droubay, C. M. Wang, and A. S. Lea, R. F. C. Farrow, L. Folks, V. Deline, and S. Anders, Appl. Phys. Lett., 82 (2003), 1257

93. D. H. Kim, J. S. Yang, K. W. Lee, S. D. Bu, and T. W. Noh,S.-J. Oh,Y.-W. Kim,J.-S. Chung,H. Tanaka, H. Y. Lee, and T. Kawai, Appl. Phys. Lett., 82 (2002), 2421

94. J.-Y. Kim, J.-H. Park, B.-G. Park, H.-J. Noh, S.-J. Oh,J. S.Yang, D.-H. Kim, S. D. Bu, T.-W. Noh, H.-J. Lin,H.-H. Hsieh, and C.T. Chen, Phys. Rev. Lett., 90 (2003), 017401-1

95. H. Kimura, T. Fukumura and M. Kawasaki K. Inaba and T. Hasegawa, H. Koinuma, Appl. Phys. Lett., 80 (2002), 94

96. T. Fukumura, Z.Jin, M. Kawasaki, T.Shono, T. Hasegawa, S. Koshihara, H. Koinuma, Appl. Phys. Lett., 78 (2001), 958

97. A. Tiwari, C. Jin, A.Kvit, D. Kumar, J.F. Muth, J. Narayan, Solid State Comm., 121 (2002), 371

98. Z. Jin, M. Murakami, T. Fukumura, y. Matsumoto, A. Ohtomo, M. Kawasaki, H. Koinuma Journ. of cryst growth, 214/215 (2000),55

99. K. Sato, H. Katayama-Yoshida, Jap. Journ.App. Phys.,39 (2000), L555 K.

100. Ando, H. Saito, Zhengwu Jin, T. Fukumura, M. Kawasaki, Y. Matsumoto, H. Koinuma, Journ.App. Phys., 89 (2001), 7284

101. S. W. Yoon, S.-B. Cho, S. C. We, S. Yoon, and B. J. Suh, H. K. Song and Y. J. Shin, Journ.App. Phys., 93 (2000), 7879

102. K. Ando, H. Saito, Zhengwu Jin, T. Fukumura, and M. Kawasaki, Y. Matsumoto, H. Koinuma, Appl. Phys. Lett., 78 (2001), 2700

103. Y. M. Cho, W.K. Choo, Appl. Phys. Lett., 80 (2002), 3358

104. Hyeon-Jun Lee and Se-Young Jeong, Chae Ryong Cho, Chul Hong Park, Appl. Phys. Lett., 81 (2002), 4020

105. W. Prellier, A. Fouchet, B. Mercey, Ch. Simon, and B. Raveau, Appl. Phys. Lett., 82 (2003), 3490

106. A. S. Risbud, N. A. Spaldin, Z. Q. Chen, S. Stemmer, and Ram Seshadri, Phys. Rev.B, 68 (2003), 205202

107. T. Fukumura, Z.Jin, A. Ohtomoto, H. Koinuma, M. Kawasaki Appl. Phys. Lett., 75 (1999), 3366

108. S. W. Jung, et al. Appl. Phys. Lett., 80 (2002), 4561

109. S. J. Han, T.H. Jang, B.G. Park, J.H. Park, Y.H. Jeong, Appl. Phys. Lett., 83 (2003), 920

110. Joseph et al. Jap. Journ. Appl. Phys., 38 (1999), L1205

111. S.W, Lim, M.C. Jeong, M-H. Ham and J-M. Myoung, Jap. Journ. Appl. Phys.,43 (2004), L280

112. S.-T. Jun, et al., J. Am. Ceram. Soc., 81 (1998), 695. 
113. S-J. Han, J. W. Song, C.-H. Yang, S. H. Park, J.-H. Park, and Y. H. Jeong, K. W. Rhie, Appl. Phys. Lett., 81 (2002), 4212

114. N. A. Spaldin, Phys. Rev. B, 69 (2004), 125201-1

115. D. P. Norton, S. J. Pearton, A. F. Hebard N. Theodoropoulou, L. A. Boatner, R. G. Wilson, Appl. Phys. Lett. , 82 (2003), 239

116. N. Theodoropoulou, G.P. Berera, V. Misra, P. Leclair, J.Philip, J.S. Moodera(unpublished)

117. Pulsed laser deposition of thin films, Eds. D.B. Chrisey and G.K. Hubler, John Wiley \& Sons, Inc. New York (1994)

118. B. D. Cullity and S.R. Stock, "Elements of X-ray diffraction", Prentice Hall, Inc. New Jersey (2001)

119. J. Guo, Int. J. of Nanotechnology,1,(2003), 231

120. A. Kotani and Shik Shin Rev. Of Modern Phys. 73, (2001), 203

121. "Encyclopedia of Materials characterization, Surface, interface, thin films", Editors: C. R. Brundle, C. A. Evans, Jr., and S. Wilson, Butterworth Heinemann (U. S. A.) 1992

122. D. Shindo, and T. Oikawa," Analytical Electron Microscopy for Materials Science", Springer-Verlag, Tokyo 2002

123. "MPMS2 Quantum Design SQUID manual.

124. "Ferromagnetic Resonance", Translated from Russian, Editor: S. V. Vonsovskii, Isreal Program for Scientific Translations, Jerusalem 1964.

125. P. Sharma, A. Gupta, F. J. Owens, A. Inoue, and K. V. Rao, J. Mag. \& Magn. Mats., (In press)

126. I. S. Jacobs, and J. S. Kouvel, Phy. Rev., 122, (1961), 412.

127. P. Sharma, A. Mansingh, and K. Sreenivas, Appl. Phys. Lett., 80(4), (2002), 553.

128. P. Sharma, S. Kumar and K. Sreenivas, J. of Mats. Res., 18(3)(2003), 545.

129. P. Sharma and K. Sreenivas, Appl. Phys. Lett., 83 (17) (2003), 3617. 